\title{
Magnetic moments of the hidden-charm pentaquark states
}

\author{
Guang-Juan Wang ${ }^{1},{ }^{*}$ Rui Chen ${ }^{2,3},{ }^{\dagger} \mathrm{Li} \mathrm{Ma}^{1},{ }^{\ddagger}$ Xiang $\mathrm{Liu}^{2,3},{ }^{\S}$ and Shi-Lin Zhu ${ }^{1,4 \pi}$ \\ ${ }^{1}$ Department of Physics and State Key Laboratory of Nuclear Physics and Technology \\ and Center of High Energy Physics, Peking University, Beijing 100871, China \\ ${ }^{2}$ Research Center for Hadron and CSR Physics, Lanzhou University and Institute of Modern Physics of CAS, Lanzhou 730000, China \\ ${ }^{3}$ School of Physical Science and Technology, Lanzhou University, Lanzhou 730000, China \\ ${ }^{4}$ Collaborative Innovation Center of Quantum Matter, Beijing 100871, China
}

(Dated: August 28, 2018)

\begin{abstract}
The magnetic moment of a baryon state is an equally important dynamical observable as its mass, which encodes crucial information of its underlying structure. According to the different color-flavor structure, we have calculated the magnetic moments of the hidden-charm pentaquark states with the isospin $\left(I, I_{3}\right)=\left(\frac{1}{2}, \frac{1}{2}\right)$ and $J^{P}=\frac{1}{2}^{ \pm}, \frac{3}{2}^{ \pm}, \frac{5}{2}^{ \pm}, \frac{7}{2}^{+}$in the molecular model, the diquark-triquark model, and the diquark-diquark-antiquark model, respectively. Although a good description for the pentaquark mass spectrum and decay patterns has been obtained in all three models, different color-flavor structures lead to different magnetic moments, which can be used to pin down their inner structures and distinguish various models.
\end{abstract}

PACS numbers: 14.20.Lq, 12.39.-x, 12.39.Mk

\section{INTRODUCTION}

The LHCb Collaboration discovered two candidates of the hidden-charm pentaquark states in the mass spectrum of $J / \psi p$ [1]. According to their decay channel, there should be five quarks $\bar{c} c u u d$ within these two $P_{c}^{+}$states. The mass and width of the heavier structure are $(4449.8 \pm 1.7 \pm 2.5) \mathrm{MeV}$ and $(39 \pm 5 \pm 19) \mathrm{MeV}$, respectively. The lighter signal has a mass $(4380 \pm 8 \pm 29)$ $\mathrm{MeV}$ and a width $(205 \pm 8 \pm 6) \mathrm{MeV}$ [1]. These two structures have opposite parity. Their $J^{P}$ quantum numbers may be $\left(\frac{3}{2}^{+}, \frac{5}{2}^{-}\right)$, $\left(\frac{3}{2}, \frac{5}{2}^{+}\right)$and $\left(\frac{5}{2}^{-}, \frac{3}{2}^{+}\right)$[1]. Several different $J^{P}$ combinations of the two states all give a good description of the experiment data. Very recently, LHCb confirms again confirmed two observed $P_{c}$ states with a model-independent analysis [2]. Before the observation of two $P_{c}$ states, the predictions of hidden-charm pentaquark were given in Refs. [3-6].

This observation has inspired theorists to explore the $P_{c}$ states extensively. Many different theoretical schemes were proposed to interpret their inner structures. An extensive review of the current experimental and theoretical progress on hidden-charm pentaquarks can be found in Ref. [7]. These studies can be categorized into four groups:

1. Molecular state scheme: since the masses of the two $P_{c}^{+}$states are close to the mass threshold of $\bar{D}^{(*)} \Sigma_{c}^{(*)}$, they are speculated to be the loosely bound molecular states of $\bar{D}^{(*)} \Sigma_{c}^{(*)}$ [8-21]. In fact, the existence of the molecular states composed of a heavy baryon and a heavy meson was predicted within the one boson exchange model before the LHCb's discovery in Ref. [3]. The hidden-charm pentaquark states were also predicted and dynamically generated with the coupled channel unitary approach in Ref. [4].

Within the framework of the molecular scheme, the mass spectrum of the pentaquark states have been calculated with QCD sum rule [10], the quark delocalization model [11], and meson exchange model [9, 14], respectively. The strong decay ratios of the pentaquark states have been calculated using heavy quark symmetry [22]. In the molecular model, the mass difference between the two $P_{c}$ states may come from $\left(m_{\Sigma_{c}^{*}}-m_{\Sigma_{c}}\right)$ and the small decay width for the heavy pentaquark states may be due to the suppression of the P-wave decay.

2. Diquark-diquark-antiqurk scheme: Besides the molecular scheme, the $P_{c}$ states could have other color-flavor configurations. For example, they were proposed as pentaquarks the diquark-diquark-antiquark $\bar{c}(c q)(q q)$ structure [23-26]. In Ref. [23], the authors suggested that the different total spin of the light diquark and the different orbital excitation leads to give the $70 \mathrm{MeV}$ mass difference of the two $P_{c}$ states. The small decay width of the heavier state is also due to the P-wave decay suppression. The mass spectrum was also calculated under this ansatz [24, 25]. In Ref. [26], the authors also calculated the week decay ratios.

\footnotetext{
*Electronic address: wgj@pku.edu.cn

$\dagger$ Electronic address: chenr2012@1zu.edu.cn

‡Electronic address: lima@pku.edu.cn

§Electronic address: xiangliu@lzu.edu.cn

"Electronic address: zhus1@pku.edu.cn
} 
3. Diquark-triquark scheme: In Ref. [27], Lebed discussed the diquark-triquark configuration of the pentaquark state and implications for their decay behavior. The mass spectrum of the $P_{c}$ states were calculated using the diquark-triqaurk model in [28], The possible partner states of these two $P_{c}$ states and their interesting decay modes were also discussed in Refs. [29-31].

4. Additionally, these two structures, especially the higher one, were also suggested to arise from the threshold effects [3234]. The $P_{c}$ states were discussed in the context of the soliton picture [35].

We notice that a good description for the pentaquark mass spectrum and decay patterns has been obtained in all three models. In other words, the mass and decay width alone can not distinguish the inner structure of the hidden-charm pentaquarks. Recall that the magnetic moment is an equally important dynamical observable of a baryon state. As shown in the following sections, the different color-flavor configurations of the $P_{c}$ states will lead to their different magnetic moments, which can be used to pin down their underlying structures.

It's the textbook knowledge that the nonrelativistic quark model is very successful in the description of the magnetic moments of the octet baryon ground states. We employ this simple framework to calculate the magnetic moments of the hidden-charm pentaquark states with different $J^{P}$ and color-flavor configurations.

In Sec. II, we first discuss the color structure of the different color-flavor configurations. In Sec. III, we calculate the magnetic moment of the $P_{c}$ states with three flavor representations in the molecular model. In Sec. IV, we calculate the $P_{c}$ magnetic moment in the diquark-diquark-antiquark model. In Sec. V, we derive the $P_{c}$ magnetic moment with the diquark-triquark model. In Sec. VI, we discuss the numerical results and summarize.

\section{COLOR CONFIGURATION OF THE $P_{c}$ STATES}

According to the color configurations, the pentaquark state may be composed of either two or three clusters. For the twocluster case, one cluster contains two quarks while the other cluster contains three quarks. According to the color structures of the two clusters, the pentaquark state may be a molecular state or a diquark-triquark state. Their possible configurations are $(\bar{c} c)\left(q_{1} q_{2} q_{3}\right),\left(\bar{c} q_{3}\right)\left(c q_{1} q_{2}\right),\left(c q_{3}\right)\left(\bar{c} q_{1} q_{2}\right)$ and $\left(q_{1} q_{2}\right)\left(\bar{c} c q_{3}\right)$. The pentaquark state may also contain three clusters: two diquarks plus an antiquark. The configuration is $\left(c q_{3}\right)\left(q_{1} q_{2}\right) \bar{c},\left(\bar{c} q_{3}\right)\left(q_{1} q_{2}\right) c$ and $(c \bar{c})\left(q_{1} q_{2}\right) q_{3}$.

For illustration, we first define the so-called color factor $I$ of the cluster which indicates whether there exists attraction within the cluster.

$$
I=\sum_{i<j, a=1}^{8} \lambda_{i}^{a} \lambda_{j}^{a}=\frac{1}{2}(C[p, q]-n C[1,0]),
$$

where $\lambda_{i}^{a}$ is the color generator of the ith quark with the cluster, $n=2,3$ for the diquark and triquark, respectively, and $C[p, q]=$ $\frac{p^{2}+p q+q^{2}}{3}+p+q$ is the first-rank Casimir operator for the cluster with the color respresentation $[p, q]$. For example, $C[1,0]=$ $C[0,1]=\frac{4}{3}$ for the quark and antiquark.

For either the $\left(\bar{c} q_{3}\right)$ or $(\bar{c} c)$ cluster within the configuration $(\bar{c} c)\left(q_{1} q_{2} q_{3}\right)$ and $\left(\bar{c} q_{3}\right)\left(q_{1} q_{2}\right)$, we have $\overline{3} \otimes 3=1 \oplus 8$. In other words, the color factor of the $\left(\bar{c} q_{3}\right)$ or $(\bar{c} c)$ cluster is $-\frac{4}{3}$ for the color singlet and $\frac{1}{6}$ for the color octet.

The color representation for the three particle cluster is $3 \otimes(3 \otimes 3)=3 \otimes(\overline{3} \oplus 6)=\left(1 \oplus 8_{2}\right) \oplus\left(10 \oplus 8_{1}\right)$ with the color factor $I_{\text {color }}=\left(-2,-\frac{1}{2}, 1,-\frac{1}{2}\right)$. To form a color singlet $P_{c}$ state, the diquark and triquark should combine as follows:

$$
\begin{aligned}
1 \otimes 1 & =1 \\
8 \otimes 8_{1} & =1 \oplus 8 \oplus 8 \oplus 10 \oplus \overline{10} \oplus 27, \\
8 \otimes 8_{2} & =1 \oplus 8 \oplus 8 \oplus 10 \oplus \overline{10} \oplus 27 .
\end{aligned}
$$

In order to form a quasibound cluster, either the $\left(\bar{c} q_{3}\right)$ or $(\bar{c} c)$ tends to be the color singlet. In other words, the $\left(\bar{c} q_{3}\right)$ forms a heavy meson and $(\bar{c} c)$ becomes a charmonium. Accordingly, the $c q_{1} q_{2}$ or $q_{1} q_{2} q_{3}$ cluster forms a color singlet baryon. Since there does not exist strong attraction between a charmonium and a light baryon, it's hard for them to form a loosely bound molecular state. We do not consider this possibility.

In contrast, the $\left(\bar{c} q_{3}\right)\left(c q_{1} q_{2}\right)$ configuration could lead to the hidden-charm molecular states composed of $\bar{D}^{(*)} \Sigma_{c}^{(*)}$, which have been studied extensively $[3,4,9,13]$. Therefore, we will consider the $\left(\bar{c} q_{3}\right)\left(c q_{1} q_{2}\right)$ structure.

For the diquark within the configurations $\left(c q_{3}\right)\left(\bar{c} q_{1} q_{2}\right)$ and $\left(q_{1} q_{2}\right)\left(\bar{c} c q_{3}\right)$, we have $3 \otimes 3=\overline{3} \oplus 6$ with the color factor $I=-\frac{2}{3}, \frac{1}{3}$, respectively. For the triquark, $\overline{3} \otimes(3 \otimes 3)=\overline{3} \otimes(\overline{3} \oplus 6)=(\overline{3} \otimes \overline{3}) \oplus(\overline{3} \otimes 6)=\left(3_{1} \oplus \overline{6}\right) \oplus\left(3_{2} \oplus 15\right)$ with the color factor $I=\frac{1}{3}(-4,-1,-4,2)$.

The triquark with the $3_{1}$ and $3_{2}$ representation tends to form a quasibound cluster with strong attraction, which then couples with the diquark in the $\overline{3}$ representation to form a color singlet pentaquark state. 
Now let us move on to the diquark-diquark-antiquark model. We note that this model converts into the molecular state if there exists one cluster with a $\bar{c}$ and a quark. The color representation of this cluster prefers the color singlet after the decomposition $3 \otimes \overline{3}=1 \oplus 8$. Then the other three quarks form another color singlet. The discussion is the same as in the case of a molecular state. In other words, the only nontrivial configuration in the diquark-diquark-antiquark model is $\left(c q_{3}\right)\left(q_{1} q_{2}\right) \bar{c}$, where both of the two diquarks are in the $\overline{3}_{c}$ representation.

In the above, we have used the color algebra to discuss the molecular configuration $\left(\bar{c} q_{3}\right)\left(c q_{1} q_{2}\right)$, the diquark-diquark-antiquark configuration $\bar{c}\left(c q_{3}\right)\left(q_{1} q_{2}\right)$ and diquark-triquark configurations $\left(c q_{3}\right)\left(\bar{c} q_{1} q_{2}\right)$ and $\left(q_{1} q_{2}\right)\left(\bar{c} c q_{3}\right)$. In the following sections, we will use these configurations to construct the color, flavor, spin and orbital wave functions of the $P_{c}$ states, which will be used in the calculation of their magnetic moments. As will be shown in the subsequent sections, different models with different color representations lead to different magnetic moments.

\section{MAGNETIC MOMENT OF THE $P_{c}$ STATES IN THE MOLECULAR SCHEME}

As a molecular state, $\bar{c} q_{3}$ and $c q_{1} q_{2}$ are in the color singlet. The two hadrons form a loosely bound molecular state. $q_{1} q_{2}$ inside the baryon should be in $\overline{3}_{c}$ representation according to Sec. II. We study the molecular state in a $S U(3)_{f} \times S U(3)_{c}$ frame. Due to the Fermi statistics, there are two different configurations for $q_{1} q_{2}$ as listed in Table I. As the heavy quark does not carry isospin number, we have only considered the three light quarks in constructing the flavor wave functions.

TABLE I: Structure of $q_{1} q_{2}$ in the baryon under the molecular frame. Here, S and A represent the wave function of the corresponding space are symmetry and antisymmetry, respectively.

$J_{\text {baryon }}=\frac{1}{2}\left|\begin{array}{ccc}\text { color } & \overline{3}_{c} & \mathrm{~A} \\
\text { space } & L=0 & \mathrm{~S} \\
\text { spin } & s=0 & \mathrm{~A} \\
\text { flavor } & \overline{3}_{f} & \mathrm{~A}\end{array}\right| J_{\text {baryon }}=\frac{1}{2}, \frac{3}{2} \mid$\begin{tabular}{lll} 
color & $\overline{3}_{c}$ & $\mathrm{~A}$ \\
space & $L=0$ & $\mathrm{~S}$ \\
spin & $s=1$ & $\mathrm{~S}$ \\
flavor & $6_{f}$ & $\mathrm{~S}$ \\
\hline
\end{tabular}

The $q_{1} q_{2}$ forms the $\overline{3}_{f}$ and $6_{f}$ flavor representation with the total spin $S=0$ and 1 , respectively. The two flavor representations are listed in Fig. 1. In Fig. 2, we illustrate the heavy baryons in the $\overline{3}_{f}$ and $6_{f}$ representation. In Table I, [ $\left.q_{1} q_{2}\right]$ forms the $\overline{3}_{f}$, which then combines with the $q_{3}$ to form the flavor representation $\overline{3}_{f} \otimes 3_{f}=1_{f} \oplus 8_{2 f}$ as illustrated in Fig. 3 . When the $q_{1} q_{2}$ is in the $6_{f}, 6_{f} \otimes 3_{f}=10_{f} \otimes 8_{1 f}$ as listed in Fig. 4. After taking into account the Clebsch-Gordan coefficients, we get the flavor wave function of the pentaquark state with the configuration $\left(\bar{c} q_{3}\right)\left(c q_{1} q_{2}\right)$. The same method is applied to the other configurations $\left(c q_{3}\right)\left(\bar{c} q_{1} q_{2}\right)$ and $\left(c q_{3}\right)\left(q_{1} q_{2}\right) \bar{c}$. The wave functions of the pentaquark states are listed in Table II.

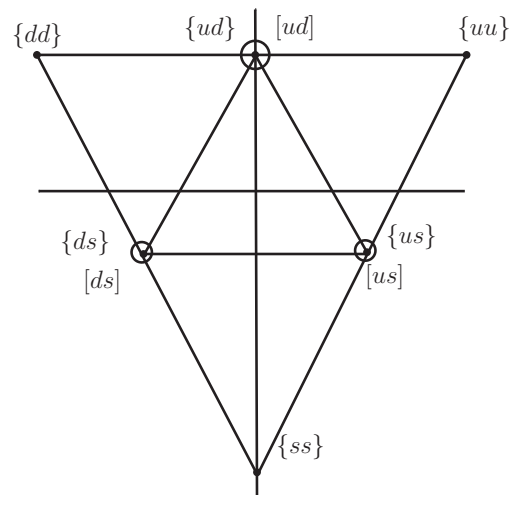

FIG. 1: The $q_{1} q_{2}$ forms the $6_{f} \oplus \overline{3}_{f}$ flavor representation. The $\left\{q_{1} q_{2}\right\}=\frac{1}{\sqrt{2}}\left(q_{1} q_{2}+q_{2} q_{1}\right)$ is for the symmetric $6_{f}$ representation, while the $\left[q_{1} q_{2}\right]=\frac{1}{\sqrt{2}}\left(q_{1} q_{2}-q_{2} q_{1}\right)$ belongs to the antisymmetric $\overline{3}_{f}$ representation. The three circles represent the $\overline{3}_{f}$ representation.

Within the molecular scheme, there is a P-wave orbital excitation in the state with positive parity. The P-wave excitation inside the baryon or meson would increase the mass of the pentaquark by $400 \mathrm{MeV}$. Moreover, a P-wave heavy hadron would be broad and unstable, which would render the pentaquark very broad. Such a possibility is not favored by the LHCb's observation. Therefore, the orbital excitation lies between the heavy meson and heavy baryon for the $P_{c}$ state with positive parity in the molecular scheme. 

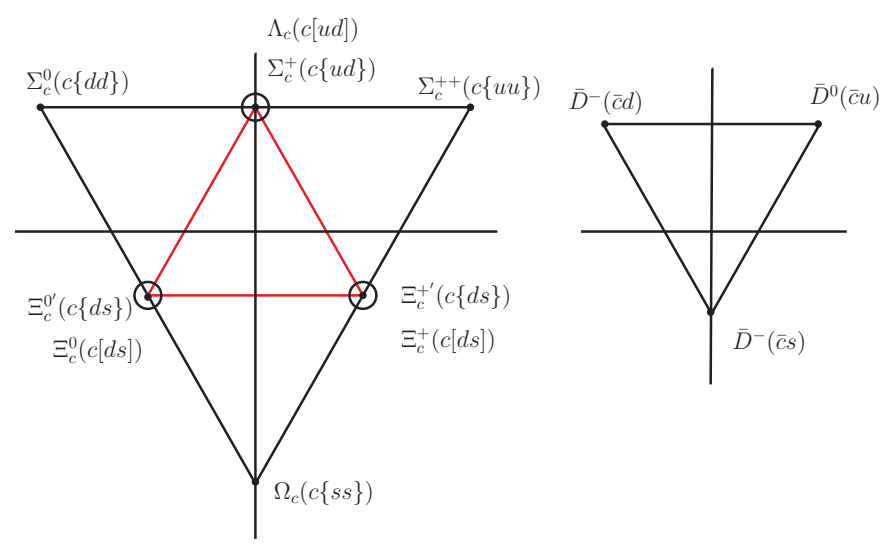

FIG. 2: The $\overline{3}_{f} \oplus 6_{f}$ and $3_{f}$ representations of the two clusters $\left(c q_{1} q_{2}\right)$ and $\left(\bar{c} q_{3}\right)$, respectively. Here, $\left[q_{1} q_{2}\right]=\frac{1}{\sqrt{2}}\left(q_{1} q_{2}-q_{2} q_{1}\right)$, $\left\{q_{1} q_{2}\right\}=$ $\frac{1}{\sqrt{2}}\left(q_{1} q_{2}+q_{2} q_{1}\right)$
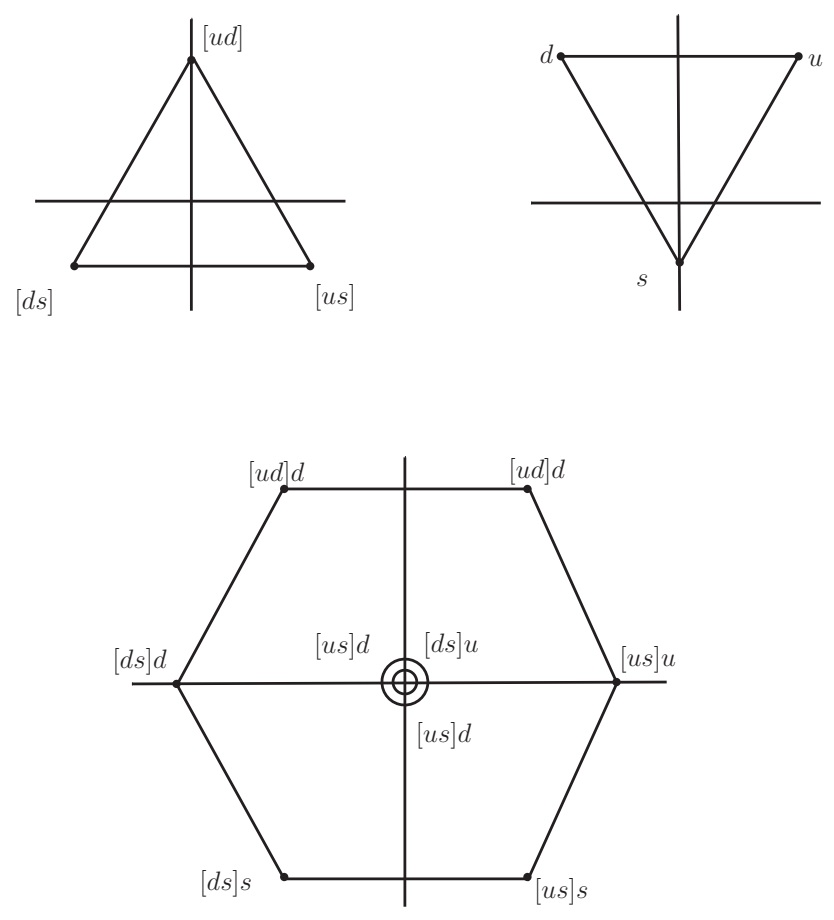

FIG. 3: The $\left[q_{1} q_{2}\right]$ in the $\overline{3}_{f}$ representation combines with the $q_{3}$ to form the flavor combination $1_{f} \oplus 8_{2 f}$. Here, $\left[q_{1} q_{2}\right]=\frac{1}{\sqrt{2}}\left(q_{1} q_{2}-q_{2} q_{1}\right)$.

The magnetic moment of the pentaquark with the configuration $\left(\bar{c} q_{3}\right)\left(c q_{1} q_{2}\right)$ is [36]

$$
\begin{aligned}
\vec{\mu} & =g_{m} \mu_{m} \vec{S}_{m}+g_{b} \mu_{b} \vec{S}_{b}+\mu_{l} \vec{l}, \\
g_{m} \mu_{m} \vec{S}_{m} & =g_{\bar{Q}} \mu_{\bar{Q}} \frac{\overrightarrow{1}}{2}+g_{q_{3}} \mu_{q_{3}} \frac{\overrightarrow{1}}{2} \\
g_{b} \mu_{b} \vec{S}_{b} & =g_{Q} \mu_{Q} \frac{\overrightarrow{1}}{2}+g_{q_{1}} \mu_{q_{1}} \frac{\overrightarrow{1}}{2}+g_{q_{2}} \mu_{q_{2}} \frac{\overrightarrow{1}}{2} \\
\mu_{l} & =\frac{m_{b} \mu_{m}+m_{m} \mu_{b}}{m_{m}+m_{b}},
\end{aligned}
$$

where $g$ is the Lande factor. The subscripts $m$ and $b$ represent meson and baryon, respectively. $l$ is the orbital excitation between the two hadrons. $Q$ and $\bar{Q}$ are the heavy quark and antiquark. $q_{1,2,3}$ is the light quark. $m_{m(b)}$ is the meson (or baryon) mass. $\mu$ 


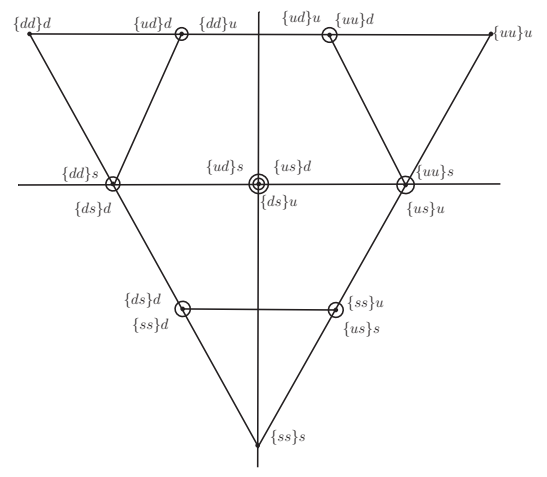

FIG. 4: The $\left\{q_{1} q_{2}\right\}$ in the $6_{f}$ representation combines with the $q_{3}$ to form the $8_{1 f} \oplus 10_{f}$. Here, $\left\{q_{1} q_{2}\right\}=\frac{1}{\sqrt{2}}\left(q_{1} q_{2}+q_{2} q_{1}\right)$. Here, the seven circles and one circle in the origin indicate the $8_{1 f}$ representation.

TABLE II: The flavor wave functions of the molecular pentaquark states with the configuration $\left(\bar{c} q_{3}\right)\left(c q_{1} q_{2}\right)$ in different representations from $3_{f} \otimes 3_{f} \otimes 3_{f}=10_{f} \oplus 8_{1 f} \oplus 8_{2 f} \oplus 1_{f}$. Here, $8_{1 f}$ and $8_{2 f}$ representation correspond to the $6_{f}$ and $\overline{3}_{f}$ representation of $q_{1} q_{2}$, respectively.

\begin{tabular}{|c|c|c|}
\hline$\left(Y, I, I_{3}\right)$ & Wave function- $8_{1 f}$ & Wave function- $8_{2 f}$ \\
\hline$\left(1, \frac{1}{2}, \frac{1}{2}\right)$ & $-\sqrt{\frac{1}{3}}(\bar{c} u)(c\{u d\})+\sqrt{\frac{2}{3}}(\bar{c} d)(c\{u u\})$ & $(\bar{c} u)(c[u d])$ \\
\hline$\left(1, \frac{1}{2},-\frac{1}{2}\right)$ & $\sqrt{\frac{1}{3}}(\bar{c} d)(c\{u d\})-\sqrt{\frac{2}{3}}(\bar{c} u)(c\{d d\})$ & $(\bar{c} d)(c[u d])$ \\
\hline$\left(-1, \frac{1}{2}, \frac{1}{2}\right)$ & $\sqrt{\frac{1}{3}}(\bar{c} s)(c\{u s\})-\sqrt{\frac{2}{3}}(\bar{c} u)(c\{s s\})$ & $(\bar{c} s)(c[u s])$ \\
\hline$\left(-1, \frac{1}{2},-\frac{1}{2}\right)$ & $\sqrt{\frac{1}{3}}(\bar{c} s)(c\{d s\})-\sqrt{\frac{2}{3}}(\bar{c} d)(c\{s s\})$ & $(\bar{c} s)(c[d s])$ \\
\hline$(0,1,1)$ & $\sqrt{\frac{1}{3}}(\bar{c} u)(c\{u s\})-\sqrt{\frac{2}{3}}(\bar{c} s)(c\{u u\})$ & $(\bar{c} u)(c[u s])$ \\
\hline$(0,1,0)$ & $\sqrt{\frac{1}{6}}[(\bar{c} d)(c\{u s\})+(\bar{c} u)(c\{d s\})]-\sqrt{\frac{2}{3}}(\bar{c} s)(c\{u d\})$ & $\frac{1}{\sqrt{2}}\{(\bar{c} d)(c[u s])+(\bar{c} u)(c[d s])\}$ \\
\hline$(0,1,-1)$ & $\sqrt{\frac{1}{3}}(\bar{c} d)(c\{d s\})-\sqrt{\frac{2}{3}}(\bar{c} s)(c\{d d\})$ & $(\bar{c} d)(c[d s])$ \\
\hline$(0,0,0)$ & $\sqrt{\frac{1}{2}}[(\bar{c} u)(c\{d s\})-(\bar{c} d)(c\{u s\})]$ & $\frac{1}{\sqrt{6}}\{(\bar{c} d)(c[u s])-(\bar{c} u)(c[d s])-2(\bar{c} s)(c[u d])\}$ \\
\hline$\left(Y, I, I_{3}\right)$ & Wave function- $10_{f}$ & Singlet \\
\hline$\left(1, \frac{3}{2}, \frac{3}{2}\right)$ & $(\bar{c} u)(c\{u u\})$ & $\frac{1}{\sqrt{3}}\{(\bar{c} d)(c[u s])-(\bar{c} u)(c[d s])+(\bar{c} s)(c[u d])\}$ \\
\hline$\left(1, \frac{3}{2}, \frac{1}{2}\right)$ & $\sqrt{\frac{2}{3}}(\bar{c} u)(c\{u d\})+\sqrt{\frac{1}{3}}(\bar{c} d)(c\{u u\})$ & \\
\hline$\left(1, \frac{3}{2},-\frac{1}{2}\right)$ & $\sqrt{\frac{2}{3}}(\bar{c} d)(c\{u d\})+\sqrt{\frac{1}{3}}(\bar{c} u)(c\{d d\})$ & \\
\hline$\left(1, \frac{3}{2},-\frac{3}{2}\right)$ & $(\bar{c} d)(c\{d d\})$ & \\
\hline$(0,1,1)$ & $\sqrt{\frac{2}{3}}(\bar{c} u)(c\{u s\})+\sqrt{\frac{1}{3}}(\bar{c} s)(c\{u u\})$ & \\
\hline$(0,1,0)$ & $\sqrt{\frac{1}{3}}[(\bar{c} d)(c\{u s\})+(\bar{c} u)(c\{d s\})]+\sqrt{\frac{1}{3}}(\bar{c} s)(c\{u d\})$ & \\
\hline$(0,1,-1)$ & $\sqrt{\frac{2}{3}}(\bar{c} d)(c\{d s\})+\sqrt{\frac{1}{3}}(\bar{c} s)(c\{d d\})$ & \\
\hline$\left(-1, \frac{1}{2}, \frac{1}{2}\right)$ & $\sqrt{\frac{1}{3}}(\bar{c} u)(c\{s s\})+\sqrt{\frac{2}{3}}(\bar{c} s)(c\{u s\})$ & \\
\hline$\left(-1, \frac{1}{2},-\frac{1}{2}\right)$ & $\sqrt{\frac{1}{3}}(\bar{c} d)(c\{s s\})+\sqrt{\frac{2}{3}}(\bar{c} s)(c\{d s\})$ & \\
\hline$(-2,0,0)$ & $(\bar{c} s)(c\{s s\})$ & \\
\hline
\end{tabular}


denotes the magnetic moment. The pentaquark state is written as

$$
\begin{gathered}
\left.\left.|J J\rangle=\mid\left[\mid\left(\left(q_{1} q_{2}\right)_{s_{12}} c_{\frac{1}{2}}\right)_{S_{b}} \otimes\left(\bar{c} q_{3}\right)_{S_{m}}\right]_{S} \otimes l\right]_{J}^{J}\right\rangle, \\
\mu=\left\langle J J\left|g_{m} \mu_{m} \vec{S}_{m}+g_{b} \mu_{b} \vec{S}_{b}+\mu_{l} \vec{l}\right| J J\right\rangle \\
=\sum_{l_{z}, S_{z}}\left\langle l l_{z}, S S_{z} \mid J J\right\rangle^{2}\left\{\mu_{l} l_{z}+\sum_{S_{z}^{\prime}}\left\langle S_{b} S_{z}^{\prime}, S_{m}\left(S_{z}-S_{z}^{\prime}\right) \mid S S_{z}\right\rangle^{2}\left[\left(S_{z}-S_{z}^{\prime}\right)\left(\mu_{\bar{Q}}+\mu_{q_{3}}\right)\right.\right. \\
\left.\left.\quad+\left\langle\frac{1}{2} \frac{1}{2}, S_{12}\left(S_{z}^{\prime}-\frac{1}{2}\right) \mid S_{b} S_{z}^{\prime}\right\rangle^{2}\left(\mu_{\bar{Q}}+\left(S_{z}^{\prime}-\frac{1}{2}\right)\left(\mu_{q_{1}}+\mu_{q_{2}}\right)\right)+\left\langle\frac{1}{2}-\frac{1}{2}, s_{12}\left(S_{z}^{\prime}+\frac{1}{2}\right) \mid S_{b} S_{z}^{\prime}\right\rangle\left(-\mu_{Q}+\left(S_{z}^{\prime}+\frac{1}{2}\right)\left(\mu_{q_{1}}+\mu_{q_{2}}\right)\right)\right]\right\},
\end{gathered}
$$

where $s_{12}$ and $S$ are the spin of the diquark inside the baryon and the spin of the pentaquark state, respectively. $l_{z}, S_{z}$, and $S_{z}^{\prime}$ are the third component of the orbital excitation, the total spin of the pentaquark state, the spin of the baryon, respectively.

The values of the constituent quark masses are

$$
m_{s}=0.5 \mathrm{GeV}, m_{u}=m_{d}=0.33 \mathrm{GeV}, m_{c}=1.55 \mathrm{GeV},
$$

which lead to the magnetic moments of the octet baryons in rough agreement with the experimental data as listed in Table III.

TABLE III: The magnetic moment of the octet baryons. The unit is the magnetic moment of the proton.

\begin{tabular}{c|c|c|c}
\hline \hline Baryons & Magnetic moment & Numerical & Experiment \\
\hline$p$ & $\frac{4}{3} \mu_{u}-\frac{1}{3} \mu_{d}$ & 2.842 & 2.793 \\
\hline$n$ & $\frac{4}{3} \mu_{d}-\frac{1}{3} \mu_{u}$ & -1.895 & -1.913 \\
\hline$\Lambda$ & $\mu_{s}$ & -0.625 & $-0.613 \pm 0.006$ \\
\hline$\Sigma^{+}$ & $\frac{4}{3} \mu_{u}-\frac{1}{3} \mu_{s}$ & 2.735 & $2.460 \pm 0.006$ \\
\hline$\Sigma^{-}$ & $\frac{4}{3} \mu_{d}-\frac{1}{3} \mu_{s}$ & -1.055 & $-1.160 \pm 0.025$ \\
\hline$\Xi^{0}$ & $\frac{4}{3} \mu_{s}-\frac{1}{3} \mu_{u}$ & -1.465 & $-1.250 \pm 0.014$ \\
\hline$\Xi^{-}$ & $\frac{4}{3} \mu_{s}-\frac{1}{3} \mu_{d}$ & -0.518 & $-0.651 \pm 0.0025$ \\
\hline$\Omega^{-}$ & $3 \mu_{s}$ & -1.876 & $-2.020 \pm 0.05$ \\
\hline \hline
\end{tabular}

We use the masses of the heavy mesons and baryons from PDG [37]. Within the molecular scheme, the $P_{c}(4380)$ and $P_{c}(4450)$ state with isospin $\frac{1}{2}$ sit right on the mass threshold of $\bar{D}^{(*)} \Sigma_{c}^{(*)}$ and belong to the $8_{1 f}$ representation since they were observed in the $J / \psi p$ channel. Their magnetic moments are listed in Table IV. The unit is the proton magnetic moment $\mu_{p}$.

The molecular states $P_{c}(4380)$ and $P_{c}(4450)$ have the flavor wave function as $-\sqrt{\frac{1}{3}} \Sigma_{c}^{+(*)} \bar{D}^{0(*)}+\sqrt{\frac{2}{3}} \Sigma_{c}^{++(*)} \bar{D}^{-(*)}$. For the molecular state composed of $\Sigma_{c} \bar{D}^{*}$ with $J^{P}=\frac{3}{2}^{+}$, there are two configurations $\left[\frac{1}{2}^{+} \otimes 1^{-}\right]_{\frac{1}{2}} \otimes 1^{-}$and $\left[\frac{1}{2}^{+} \otimes 1^{-}\right]_{\frac{3}{2}} \otimes 1^{-}$. Their magnetic moments are quite different, i.e. $-0.740 \mu_{p}$ and $1.041 \mu_{p}$, respectively. Therefore, the study of the magnetic moment can help probe the inner structures of the $P_{c}$ state. For the $\Sigma_{c}^{*} \bar{D}^{*}$ state with $J^{P}=\frac{3}{2}^{ \pm}, \frac{5}{2}^{ \pm}$, the magnetic moments are around 2. For example, the $\Sigma_{c}^{*} \bar{D}^{*}$ state with $J^{P}=\frac{3}{2}^{+}$has three different configurations, $\left.\left[\frac{3}{2}^{+} \otimes 1^{-}\right]_{\frac{1}{2}} \otimes 1^{-}, \frac{3}{2}^{+} \otimes 1^{-}\right]_{\frac{3}{2}} \otimes 1^{-}$and $\left[\frac{3}{2}^{+} \otimes 1^{-}\right]_{\frac{5}{2}} \otimes 1^{-}$. Their magnetic moments are $2.040 \mu_{p}, 1.668 \mu_{p}$ and $2.326 \mu_{p}$, respectively.

\section{MAGNETIC MOMENT OF THE $P_{c}$ STATES IN THE DIQUARK-DIQUARK-ANTIQUARK MODEL}

The diquark-diquark-antiquark configuration is $\left(c q_{3}\right)\left(q_{1} q_{2}\right) \bar{c}$. The magnetic moment of the $P_{c}$ state with positive parity depends on the position of the P-wave excitation. Similar to the P-wave light baryon, there are two P-wave excitation modes inside 
TABLE IV: The magnetic moment of the molecular pentaquark states $\left(\bar{c} q_{3}\right)\left(c q_{1} q_{2}\right)$ with the isospin $\left(I, I_{3}\right)=\left(\frac{1}{2}, \frac{1}{2}\right)$ in the $8_{1 f}$ flavor representation from $6_{f} \otimes 3_{f}=10_{f} \oplus 8_{1 f}$. The third line denotes the $J^{P}$ of the heavy baryon, meson, and orbital excitation. The unit is the magnetic moment of the proton.

\begin{tabular}{|c|c|c|c|c|c|c|c|}
\hline \multirow[b]{2}{*}{$\left(Y, I, I_{3}\right)$} & \multicolumn{3}{|c|}{${ }^{2} S_{\frac{1}{2}}\left(J^{P}=\frac{1}{2}^{-}\right)$} & \multicolumn{3}{|c|}{${ }^{4} S_{\frac{3}{2}}\left(J^{P}=\frac{3}{2}^{-}\right)$} & \multirow{2}{*}{$\begin{array}{l}{ }^{6} S_{\frac{5}{2}}^{-}\left(J^{P}=\frac{5}{2}^{-}\right) \\
\frac{3}{2}^{+} \otimes 1^{-} \otimes 0^{+}\end{array}$} \\
\hline & $\frac{1}{2}^{+} \otimes 0^{-} \otimes 0^{+}$ & $\frac{1}{2}^{+} \otimes 1^{-} \otimes 0^{+}$ & $\frac{3}{2}^{+} \otimes 1^{-} \otimes 0^{+}$ & $\frac{1}{2}^{+} \otimes 1^{-} \otimes 0^{+}$ & $\frac{3}{2}^{+} \otimes 0^{-} \otimes 0^{+}$ & $\frac{3}{2}^{+} \otimes 1^{-} \otimes 0^{+}$ & \\
\hline \multirow[t]{2}{*}{$\left(1, \frac{1}{2}, \frac{1}{2}\right)$} & 1.760 & -0.856 & 1.938 & 1.357 & 3.246 & 2.219 & 2.842 \\
\hline & \multicolumn{2}{|c|}{${ }^{2} P_{\frac{1}{2}}\left(J^{P}=\frac{1}{2}^{+}\right)$} & \multicolumn{2}{|c|}{${ }^{4} P_{\frac{1}{2}}\left(J^{P}=\frac{1}{2}^{+}\right)$} & ${ }^{2} P_{\frac{1}{2}}\left(J^{P}=\frac{1}{2}^{+}\right)$ & ${ }^{4} P_{\frac{1}{2}}\left(J^{P}=\frac{1}{2}^{+}\right)$ & \\
\hline$\left(Y, I, I_{3}\right)$ & $\frac{1}{2}^{+} \otimes 0^{-} \otimes 1^{-}$ & {$\left[\frac{1}{2}^{+} \otimes 1^{-}\right]_{\frac{1}{2}} \otimes 1^{-}$} & {$\left[\frac{1}{2}^{+} \otimes 1^{-}\right]_{\frac{3}{2}} \otimes 1^{-}$} & $\frac{3}{2}^{+} \otimes 0^{-} \otimes 1^{-}$ & {$\left[\frac{3}{2}^{+} \otimes 1^{-}\right]_{\frac{1}{2}} \otimes 1^{-}$} & {$\left[\frac{3}{2}^{+} \otimes 1^{-}\right]_{\frac{3}{2}} \otimes 1^{-}$} & \\
\hline \multirow[t]{2}{*}{$\left(1, \frac{1}{2}, \frac{1}{2}\right)$} & -0.530 & 0.363 & 0.715 & 1.779 & -0.578 & 1.199 & \\
\hline & \multicolumn{2}{|c|}{${ }^{2} P_{\frac{3}{2}}\left(J^{P}=\frac{3}{2}{ }^{+}\right)$} & \multicolumn{2}{|c|}{${ }^{4} P_{\frac{3}{2}}\left(J^{P}=\frac{3}{2}{ }^{+}\right)$} & ${ }^{2} P_{\frac{3}{2}}\left(J^{P}=\frac{3}{2}^{+}\right)$ & ${ }^{4} P_{\frac{3}{2}}\left(J^{P}=\frac{3}{2}{ }^{+}\right)$ & ${ }^{6} P_{\frac{3}{2}}\left(J^{P}=\frac{3}{2}{ }^{+}\right)$ \\
\hline$\left(Y, I, I_{3}\right)$ & $\frac{1}{2}^{+} \otimes 0^{-} \otimes 1^{-}$ & {$\left[\frac{1}{2}^{+} \otimes 1^{-}\right]_{\frac{1}{2}} \otimes 1^{-}$} & {$\left[\frac{1}{2}^{+} \otimes 1^{-}\right]_{\frac{3}{2}} \otimes 1^{-}$} & $\frac{3}{2}^{+} \otimes 0^{-} \otimes 1^{-}$ & {$\left[\frac{3}{2}^{+} \otimes 1^{-}\right]_{\frac{1}{2}} \otimes 1^{-}$} & {$\left[\frac{3}{2}^{+} \otimes 1^{-}\right]_{\frac{3}{2}} \otimes 1^{-}$} & {$\left[\frac{3}{2}^{+} \otimes 1^{-}\right]_{\frac{5}{2}} \otimes 1^{-}$} \\
\hline \multirow[t]{2}{*}{$\left(1, \frac{1}{2}, \frac{1}{2}\right)$} & 1.846 & -0.740 & 1.041 & 2.409 & 2.040 & 1.668 & 2.326 \\
\hline & \multicolumn{3}{|c|}{${ }^{4} P_{\frac{5}{2}}\left(J^{P}=\frac{5}{2}^{+}\right)$} & ${ }^{6} P_{\frac{5}{2}}\left(J^{P}=\frac{5}{2}^{+}\right)$ & ${ }^{6} P_{\frac{7}{2}}\left(J^{P}=\frac{7}{2}^{+}\right)$ & & \\
\hline$\left(Y, I, I_{3}\right)$ & $\frac{1}{2}^{+} \otimes 1^{-} \otimes 1^{-}$ & $\frac{3}{2}^{+} \otimes 0^{-} \otimes 1^{-}$ & {$\left[\frac{3}{2}^{+} \otimes 1^{-}\right]_{\frac{3}{2}} \otimes 1^{-}$} & {$\left[\frac{3}{2}^{+} \otimes 1^{-}\right]_{\frac{5}{2}} \otimes 1^{-}$} & $\frac{3}{2}^{+} \otimes 1^{-} \otimes 1^{-}$ & & \\
\hline$\left(1, \frac{1}{2}, \frac{1}{2}\right)$ & 1.473 & 3.319 & 2.322 & 2.547 & 2.945 & & \\
\hline
\end{tabular}

the three-body bound state, the $\rho$ and the $\lambda$ excitation. The $\rho$ mode P-wave orbital excitation lies between the diquark $c q_{3}$ and diquark $q_{1} q_{2}$. The $\lambda$ mode P-wave orbital excitation lies between the $\bar{c}$ and the center of mass system of the $c q_{3}$ and $q_{1} q_{2}$. Now the pentaquark state $|J J\rangle$ is written as

$$
|J J\rangle=\left|\left[\left[\left[\left(c q_{3}\right)_{s_{1}} \otimes\left(q_{1} q_{2}\right)_{s_{2}}\right]_{s^{\prime}} \otimes \bar{c}_{\frac{1}{2}}\right]_{S} \otimes l\right]_{J}^{J}\right\rangle
$$

where $s_{1}$ and $s_{2}$ are the spin of the diquark $c q_{3}$ and $q_{1} q_{2}$, respectively. They two couple into the spin $s^{\prime}$, which combines with the spin of the $\bar{c}$ to form the total spin of the pentaquark state $S . l$ is the orbital excitation. $J$ is the total angular momentum. The magnetic moment of the pentaquark state is

$$
\begin{aligned}
\mu= & \left\langle J J\left|g_{1} \mu_{1} \vec{S}_{1}+g_{2} \mu_{2} \vec{S}_{2}+g_{\bar{Q}} \mu_{\bar{Q}} \frac{\overrightarrow{1}}{2}+\mu_{l} \vec{l}\right| J J\right\rangle \\
= & \sum_{m, S_{z}}\left\langle l m, S S_{z} \mid J J\right\rangle^{2}\left\{\mu_{l} m+\sum_{S_{z}^{\prime}}\left\langle S^{\prime} S_{z}^{\prime} \frac{1}{2}\left(S_{z}-S_{z}^{\prime}\right) \mid S S_{z}\right\rangle^{2}\left[2\left(S_{z}-S_{z}^{\prime}\right) \mu_{\bar{Q}}\right.\right. \\
& \left.\left.+\sum_{S_{1 z}}\left\langle S_{1} S_{1 z} S_{2}\left(S_{z}^{\prime}-S_{1 z}\right) \mid S^{\prime} S_{z}^{\prime}\right\rangle^{2}\left(S_{1 z}\left(\mu_{Q}+\mu_{q 3}\right)+\left(S_{z}^{\prime}-S_{1 z}\right)\left(\mu_{q 1}+\mu_{q 2}\right)\right)\right]\right\},
\end{aligned}
$$

where $m, S_{z}$, and $S_{z}^{\prime}$ are the third component of the orbital excitation, the total spin of the pentaquark state, and the diquark spin, respectively. We use the values of the diquark masses from Ref. [38]

$$
\begin{aligned}
& m_{[q q]}=0.710 \mathrm{GeV}, m_{\{q q\}}=0.909 \mathrm{GeV}, m_{[q s]}=0.948 \mathrm{GeV}, m_{\{q s\}}=1.069 \mathrm{GeV}, m_{\{s s\}}=1.203 \mathrm{GeV}, \\
& m_{[c q]}=1.937 \mathrm{GeV}, m_{\{c q\}}=2.036 \mathrm{GeV}, m_{[c s]}=2.091 \mathrm{GeV}, m_{\{c s\}}=2.158 \mathrm{GeV} .
\end{aligned}
$$

We list the flavor wave functions of the pentaquark states in this model in Table V. The analytic expressions for magnetic moments of the configurations $\left(c q_{3}\right)\left(q_{1} q_{2}\right) \bar{c}$ in the $8_{2 f}$ and $8_{1 f}$ flavor representations are in Table VI. In Tables VII and VIII, we list the magnetic moments of the pentaquark states with the isospin $\left(I, I_{3}\right)=\left(\frac{1}{2}, \frac{1}{2}\right)$. The states with the positive parities have the $\rho$ and $\lambda$ excitation modes in the two tables, respectively.

The numerical results for the $\frac{5}{2}^{-}$pentaquark states in $8_{1 f}$ representation are the same as those in the molecular model and diquark-diquark-antiquark model, which is illustrated in Tables IV, VII, and VIII. The magnetic moments of the $\frac{5}{2}^{-}$pentaquark states in the diquark-triquark scheme are also the same as those in the above two models, which can be seen in Sec. V. For the 
TABLE V: The flavor wave functions of the pentaquark states in the diquark-diquark-antiquark model with the configuration $\left(c q_{3}\right)\left(q_{1} q_{2}\right) \bar{c}$ in different representations from $3_{f} \otimes 3_{f} \otimes 3_{f}=10_{f} \oplus 8_{1 f} \oplus 8_{2 f} \oplus 1_{f}$. Here, $8_{1 f}$ and $8_{2 f}$ representation correspond to the representation for $q_{1} q_{2}$ being $6_{f}$ and $\overline{3}_{f}$, respectively.

\begin{tabular}{|c|c|c|}
\hline$\left(Y, I, I_{3}\right)$ & Wave function- $8_{1 f}$ & Wave function- $8_{2 f}$ \\
\hline$\left(1, \frac{1}{2}, \frac{1}{2}\right)$ & $-\sqrt{\frac{1}{3}}(c u)\{u d\} \bar{c}+\sqrt{\frac{2}{3}}(c d)\{u u\} \bar{c}$ & $(c u)[u d] \bar{c}$ \\
\hline$\left(1, \frac{1}{2},-\frac{1}{2}\right)$ & $\sqrt{\frac{1}{3}}(c d) c\{u d\} \bar{c}-\sqrt{\frac{2}{3}}(c u)\{d d\} \bar{c}$ & $(c d)[u d] \bar{c}$ \\
\hline$(0,1,1)$ & $\sqrt{\frac{1}{3}}(c u)\{u s\} \bar{c}-\sqrt{\frac{2}{3}}(c s)\{u u\} \bar{c}$ & $(c u)[u s] \bar{c}$ \\
\hline$(0,1,0)$ & $\sqrt{\frac{1}{6}}[(c d)\{u s\} \bar{c}+(c u)\{d s\} \bar{c}]-\sqrt{\frac{2}{3}}(c s)\{u d\} \bar{c}$ & $\frac{1}{\sqrt{2}}\{(c d)[u s] \bar{c}+(c u)[d s] \bar{c}\}$ \\
\hline$(0,1,-1)$ & $\sqrt{\frac{1}{3}}(c d)\{d s\} \bar{c}-\sqrt{\frac{2}{3}}(c s)\{d d\} \bar{c}$ & $(c d)[d s] \bar{c}$ \\
\hline$(0,0,0)$ & $\sqrt{\frac{1}{2}}[(c u)\{d s\} \bar{c}-(c d)\{u s\} \bar{c}]$ & $\frac{1}{\sqrt{6}}\{(c d)[u s] \bar{c}-(c u)[d s] \bar{c}-2(c s)[u d] \bar{c}\}$ \\
\hline$\left(-1, \frac{1}{2}, \frac{1}{2}\right)$ & $\sqrt{\frac{1}{3}}(c s)\{u s\} \bar{c}-\sqrt{\frac{2}{3}}(c u)\{s s\} \bar{c}$ & $(c s)[u s] \bar{c}$ \\
\hline$\left(-1, \frac{1}{2},-\frac{1}{2}\right)$ & $\sqrt{\frac{1}{3}}(c s)\{d s\} \bar{c}-\sqrt{\frac{2}{3}}(c d)\{s s\} \bar{c}$ & $(c s)[d s] \bar{c}$ \\
\hline$\left(Y, I, I_{3}\right)$ & Wave function- $10_{f}$ & Singlet \\
\hline$\left(1, \frac{3}{2}, \frac{3}{2}\right)$ & $(c u)\{u u\} \bar{c}$ & $\frac{1}{\sqrt{3}}\{(c d[u s]) \bar{c}-(c u)[d s] \bar{c}+(c s)[u d] \bar{c}\}$ \\
\hline$\left(1, \frac{3}{2}, \frac{1}{2}\right)$ & $\sqrt{\frac{2}{3}}(c u)\{u d\} \bar{c}+\sqrt{\frac{1}{3}}(c d)\{u u\} \bar{c}$ & \\
\hline$\left(1, \frac{3}{2},-\frac{1}{2}\right)$ & $\sqrt{\frac{2}{3}}(c d)\{u d\} \bar{c}+\sqrt{\frac{1}{3}}(c u)\{d d\} \bar{c}$ & \\
\hline$\left(1, \frac{3}{2},-\frac{3}{2}\right)$ & $(c d)(\{d d\}) \bar{c}$ & \\
\hline$(0,1,1)$ & $\sqrt{\frac{2}{3}}(c u)\{u s\} \bar{c}+\sqrt{\frac{1}{3}}(c s)\{u u\} \bar{c}$ & \\
\hline$(0,1,0)$ & $\sqrt{\frac{1}{3}}[(c d)\{u s\} \bar{c}+(c u)\{d s\} \bar{c}]+\sqrt{\frac{1}{3}}(c s)\{u d\} \bar{c}$ & \\
\hline$(0,1,-1)$ & $\sqrt{\frac{2}{3}}(c d)\{d s\} \bar{c}+\sqrt{\frac{1}{3}}(c s)\{d d\} \bar{c}$ & \\
\hline$\left(-1, \frac{1}{2}, \frac{1}{2}\right)$ & $\sqrt{\frac{1}{3}}(c u)\{s s\} \bar{c}+\sqrt{\frac{2}{3}}(c s)\{u s\} \bar{c}$ & \\
\hline$\left(-1, \frac{1}{2},-\frac{1}{2}\right)$ & $\sqrt{\frac{1}{3}}(c d)\{s s\} \bar{c}+\sqrt{\frac{2}{3}}(c s)\{d s\} \bar{c}$ & \\
\hline$(-2,0,0)$ & $(c s)\{s s\} \bar{c}$ & \\
\hline
\end{tabular}

$\frac{5}{2}^{-}$pentaquark states, the analytic formulas for the magnetic moments are the same in different models as illustrated in Tables VI and XII. The sum of the magnetic moments of all the constituent quarks leads to those of the pentaquark states. Therefore, the magnetic moments of the $\frac{5}{2}^{-}$only depend on the flavor content and do not dependent on their configurations and the models.

The $P_{c}(4380)$ and $P_{c}(4450)$ with the isospin $\left(I, I_{3}\right)=\left(\frac{1}{2}, \frac{1}{2}\right)$ are in the $8_{1 f}$ or $8_{2 f}$ representation in the diquark-diquarkantiquark scheme. The numerical results for the states with the $\rho$ and $\lambda$ excitation modes are different. The magnetic moments of the $P_{c}$ states in the $8_{1 f}$ representation with $J^{P}=\frac{3}{2}^{+}, \frac{5}{2}^{+}$and $\lambda$ excitation are larger than those with the $\rho$ excitation except the configuration $\left(\left(1^{+} \otimes 1^{+}\right)_{2} \otimes \frac{1}{2}\right)_{\frac{5}{2}} \otimes 1^{-}$, as illustrated in Tables VII and VIII.

\section{MAGNETIC MOMENT OF THE $P_{c}$ STATES IN THE DIQUARK-TRIQUARK MODEL}

The diquark-triquark configurations are either $\left(c q_{3}\right)\left(\bar{c} q_{1} q_{2}\right)$ or $\left(q_{1} q_{2}\right)\left(\bar{c} c q_{3}\right)$. Both the $\left(c q_{3}\right)$ diquark within $\left(c q_{3}\right)\left(\bar{c} q_{1} q_{2}\right)$ and $\left(q_{1} q_{2}\right)$ diquark within $\left(q_{1} q_{2}\right)\left(\bar{c} c q_{3}\right)$ are in a $\overline{3}_{c}$ representation. The triquark $\bar{c} q_{1} q_{2}$ or $\left(\bar{c} c q_{3}\right)$ is also in the $3_{c}$ representation.

However, as pointed out in Sec II, the diquark $q_{1} q_{2}$ in the triquark $\left(\bar{c} q_{1} q_{2}\right)$ can be either in a $\overline{3}_{c}$ or $6_{c}$ representation. When the $q_{1} q_{2}$ is in the $\overline{3}_{c}$ representation, the structures are the same as that in Table I. Their wave functions are listed in Table IX. When the $q_{1} q_{2}$ is in the $6_{c}$ representation, the structures are listed in Table $\mathrm{X}$. 
TABLE VI: The analytic formula for the magnetic moment of the pentaquark in the configuration $\left(Q q_{3}\right)\left(q_{1} q_{2}\right) \bar{Q}$ when $s_{q_{1} q_{2}}=0$ and 1 .

\begin{tabular}{|c|c|c|c|}
\hline \multicolumn{4}{|r|}{$s_{q_{1} q_{2}}=0$} \\
\hline$J^{P}$ & ${ }^{2 s+1} L_{J}$ & & Formula \\
\hline$\frac{1}{2}^{-}$ & ${ }^{2} S_{\frac{1}{2}}$ & $\begin{array}{l}0^{+} \otimes 0^{+} \otimes \frac{1}{2}^{-} \otimes 0^{+} \\
1^{+} \otimes 0^{+} \otimes \frac{1}{2}^{-} \otimes 0^{+}\end{array}$ & $\begin{array}{c}\mu_{\bar{Q}} \\
\frac{1}{3}\left(2 \mu_{Q}+2 \mu_{q_{3}}-\mu_{\bar{Q}}\right) \\
\end{array}$ \\
\hline$\frac{3}{2}^{-}$ & ${ }^{4} S_{\frac{3}{2}}$ & $1^{+} \otimes 0^{+} \otimes \frac{1}{2}^{-} \otimes 0^{+}$ & $\mu_{Q}+\mu_{q_{3}}+\mu_{\bar{Q}}$ \\
\hline \multirow[t]{2}{*}{$\frac{1}{2}^{+}$} & ${ }^{2} P_{\frac{1}{2}}$ & $\begin{array}{c}0^{+} \otimes 0^{+} \otimes \frac{1}{2}^{-} \otimes 1^{-} \\
\left(1^{+} \otimes 0^{+} \otimes \frac{1}{2}^{-}\right)_{\frac{1}{2}} \otimes 1^{-}\end{array}$ & $\begin{array}{c}\frac{1}{3}\left(2 \mu_{1}-\mu_{\bar{Q}}\right) \\
\frac{1}{9}\left(6 \mu_{1}-2 \mu_{Q}-2 \mu_{q_{3}}+\mu_{\bar{Q}}\right)\end{array}$ \\
\hline & ${ }^{4} P_{\frac{1}{2}}$ & $\left(1^{+} \otimes 0^{+} \otimes \frac{1}{2}^{-}\right) \frac{3}{2}^{-} \otimes 1^{-}$ & $\frac{1}{9}\left(-3 \mu_{1}+5\left(\mu_{Q}+\mu_{q_{3}}+\mu_{\bar{Q}}\right)\right)$ \\
\hline \multirow[t]{2}{*}{$\frac{3}{2}^{+}$} & ${ }^{2} P_{\frac{3}{2}}$ & $\begin{array}{c}0^{+} \otimes 0^{+} \otimes \frac{1}{2}^{-} \otimes 1^{-} \\
\left(1^{+} \otimes 0^{+} \otimes \frac{1}{2}^{-}\right)_{\frac{1}{2}} \otimes 1^{-}\end{array}$ & $\begin{array}{c}\mu_{1}+\mu_{\bar{Q}} \\
\frac{1}{3}\left(3 \mu_{1}+2 \mu_{Q}+2 \mu_{q_{3}}-\mu_{\bar{Q}}\right) \\
\end{array}$ \\
\hline & ${ }^{4} P_{\frac{3}{2}}$ & $\left(1^{+} \otimes 0^{+} \otimes \frac{1}{2}^{-}\right)_{\frac{3}{2}} \otimes 1^{-}$ & $\frac{1}{15}\left(6 \mu_{1}+11\left(\mu_{Q}+\mu_{q_{3}}+\mu_{\bar{Q}}\right)\right)$ \\
\hline$\frac{5}{2}^{+}$ & ${ }^{4} P_{\frac{5}{2}}$ & $1^{+} \otimes 0^{+} \otimes \frac{1}{2}^{-} \otimes 1^{-}$ & $\mu_{1}+\mu_{Q}+\mu_{q_{3}}+\mu_{\bar{Q}}$ \\
\hline \multicolumn{4}{|r|}{$s_{q_{1} q_{2}}=1$} \\
\hline$J^{P}$ & ${ }^{2 s+1} L_{J}$ & & Formula \\
\hline$\frac{1}{2}^{-}$ & ${ }^{2} S_{\frac{1}{2}}$ & $\begin{array}{c}0^{+} \otimes 1^{+} \otimes \frac{1}{2}^{-} \otimes 0^{+} \\
\left(1^{+} \otimes 1^{+}\right)_{0} \otimes \frac{1}{2}^{-} \otimes 0^{+} \\
\left(1^{+} \otimes 1^{+}\right)_{1} \otimes \frac{1}{2}^{-} \otimes 0^{+}\end{array}$ & $\begin{array}{c}\frac{1}{3}\left(2 \mu_{q_{1}}+2 \mu_{q_{2}}-\mu_{\bar{Q}}\right) \\
\mu_{\bar{Q}} \\
\frac{1}{3}\left(\mu_{Q}+\mu_{q_{1}}+\mu_{q_{2}}+\mu_{q_{3}}-\mu_{\bar{Q}}\right) \\
\end{array}$ \\
\hline$\frac{3}{2}^{-}$ & ${ }^{4} S_{\frac{3}{2}}$ & $\begin{array}{l}\left(0^{+} \otimes 1^{+}\right) \otimes \frac{1}{2}^{-} \otimes 0^{+} \\
\left(1^{+} \otimes 1^{+}\right)_{1} \otimes \frac{1}{2}^{-} \otimes 0^{+} \\
\left(1^{+} \otimes 1^{+}\right)_{2} \otimes \frac{1}{2}^{-} \otimes 0^{+}\end{array}$ & $\begin{array}{c}\mu_{q_{1}}+\mu_{q_{2}}+\mu_{\bar{Q}} \\
\frac{1}{2}\left(\mu_{Q}+\mu_{q_{1}}+\mu_{q_{2}}+\mu_{q_{3}}+2 \mu_{\bar{Q}}\right) \\
\frac{3}{10}\left(3 \mu_{Q}+3 \mu_{q_{1}}+3 \mu_{q_{2}}+3 \mu_{q_{3}}-2 \mu_{\bar{Q}}\right)\end{array}$ \\
\hline$\frac{5}{2}^{-}$ & ${ }^{6} S_{\frac{5}{2}}$ & $1^{+} \otimes 1^{+} \otimes \frac{1}{2}^{-} \otimes 0^{+}$ & $\mu_{Q}+\mu_{q_{1}}+\mu_{q_{2}}+\mu_{q_{3}}+\mu_{\bar{Q}}$ \\
\hline \multirow[t]{2}{*}{$\frac{1}{2}^{+}$} & ${ }^{2} P_{\frac{1}{2}}$ & $\begin{array}{l}\left(0^{+} \otimes 1^{+} \otimes \frac{1}{2}^{-}\right)_{\frac{1}{2}} \otimes 1^{-} \\
\left(\left(1^{+} \otimes 1^{+}\right)_{0} \otimes \frac{1}{2}^{-}\right)_{\frac{1}{2}} \otimes 1^{-} \\
\left(\left(1^{+} \otimes 1^{+}\right)_{1} \otimes \frac{1}{2}^{-}\right)_{\frac{1}{2}} \otimes 1^{-}\end{array}$ & $\begin{array}{c}\frac{1}{9}\left(6 \mu_{1}-2 \mu_{q_{1}}-2 \mu_{q_{2}}+\mu_{\bar{Q}}\right) \\
\frac{1}{3}\left(2 \mu_{1}-\mu_{\bar{Q}}\right) \\
\frac{1}{9}\left(6 \mu_{1}-\mu_{Q}-\mu_{q_{1}}-\mu_{q_{2}}-\mu_{q_{3}}+\mu_{\bar{Q}}\right) \\
\end{array}$ \\
\hline & ${ }^{4} P_{\frac{1}{2}}$ & $\begin{array}{l}\left(0^{+} \otimes 1^{+} \otimes \frac{1}{2}^{-}\right)_{\frac{3}{2}} \otimes 1^{-} \\
\left(\left(1^{+} \otimes 1^{+}\right)_{1} \otimes \frac{1}{2}^{-}\right)_{\frac{3}{2}} \otimes 1^{-} \\
\left(\left(1^{+} \otimes 1^{+}\right)_{2} \otimes \frac{1}{2}^{-}\right)_{\frac{3}{2}} \otimes 1^{-}\end{array}$ & $\begin{array}{c}\frac{1}{9}\left(-3 \mu_{1}+5\left(\mu_{q_{1}}+\mu_{q_{2}}+\mu_{\bar{Q}}\right)\right) \\
\frac{1}{18}\left(-6 \mu_{1}+5\left(\mu_{Q}+\mu_{q_{1}}+\mu_{q_{2}}+\mu_{q_{3}}+2 \mu_{\bar{Q}}\right)\right) \\
\frac{1}{6}\left(-2 \mu_{1}+3 \mu_{Q}+3 \mu_{q_{1}}+3 \mu_{q_{2}}+3 \mu_{q_{3}}-2 \mu_{\bar{Q}}\right)\end{array}$ \\
\hline \multirow[t]{3}{*}{$\frac{3}{2}^{+}$} & ${ }^{2} P_{\frac{3}{2}}$ & $\begin{array}{l}\left(0^{+} \otimes 1^{+} \otimes \frac{1}{2}^{-}\right)_{\frac{1}{2}} \otimes 1^{-} \\
\left(\left(1^{+} \otimes 1^{+}\right)_{0} \otimes \frac{1}{2}^{-}\right)_{\frac{1}{2}} \otimes 1^{-} \\
\left(\left(1^{+} \otimes 1^{+}\right)_{1} \otimes \frac{1}{2}^{-}\right)_{\frac{1}{2}} \otimes 1^{-}\end{array}$ & $\begin{array}{c}\frac{1}{3}\left(3 \mu_{1}+2 \mu_{q_{1}}+2 \mu_{q_{2}}-\mu_{\bar{Q}}\right) \\
\mu_{1}+\mu_{\bar{Q}} \\
\frac{1}{3}\left(3 \mu_{1}+\mu_{Q}+\mu_{q_{1}}+\mu_{\mathrm{q} 2}+\mu_{q_{3}}-\mu_{\bar{Q}}\right)\end{array}$ \\
\hline & ${ }^{4} P_{\frac{3}{2}}$ & $\begin{array}{l}\left(0^{+} \otimes 1^{+} \otimes \frac{1}{2}^{-}\right)_{\frac{3}{2}} \otimes 1^{-} \\
\left(\left(1^{+} \otimes 1^{+}\right)_{1} \otimes \frac{1}{2}^{-}\right)_{\frac{3}{2}} \otimes 1^{-} \\
\left(\left(1^{+} \otimes 1^{+}\right)_{2} \otimes \frac{1}{2}^{-}\right)_{\frac{3}{2}} \otimes 1^{-}\end{array}$ & $\begin{array}{c}\frac{1}{15}\left(6 \mu_{1}+11\left(\mu_{q_{1}}+\mu_{q_{2}}+\mu_{\bar{Q}}\right)\right) \\
\frac{1}{30}\left(12 \mu_{1}+11\left(\mu_{Q}+\mu_{q_{1}}+\mu_{q_{2}}+\mu_{q_{3}}+2 \mu_{\bar{Q}}\right)\right) \\
\frac{1}{50}\left(20 \mu_{1}+11\left(3 \mu_{Q}+3 \mu_{q_{1}}+3 \mu_{\mathrm{q} 2}+3 \mu_{q_{3}}-2 \mu_{\bar{Q}}\right)\right)\end{array}$ \\
\hline & ${ }^{6} P_{\frac{3}{2}}$ & $\left(\left(1^{+} \otimes 1^{+}\right)_{2} \otimes \frac{1}{2}^{-}\right)_{\frac{5}{2}} \otimes 1^{-}$ & $\frac{3}{25}\left(-5 \mu_{1}+7\left(\mu_{Q}+\mu_{q_{1}}+\mu_{q_{2}}+\mu_{q_{3}}+\mu_{\bar{Q}}\right)\right)$ \\
\hline \multirow[t]{2}{*}{$\frac{5}{2}^{+}$} & ${ }^{4} P_{\frac{5}{2}}$ & $\begin{array}{l}\left(0^{+} \otimes 1^{+} \otimes \frac{1}{2}^{-}\right) \otimes 1^{-} \\
\left(\left(1^{+} \otimes 1^{+}\right)_{1} \otimes \frac{1}{2}^{-}\right)_{\frac{3}{2}} \otimes 1^{-} \\
\left(\left(1^{+} \otimes 1^{+}\right)_{2} \otimes \frac{1}{2}^{-}\right)_{\frac{3}{2}} \otimes 1^{-}\end{array}$ & $\begin{array}{c}\mu_{1}+\mu_{q_{1}}+\mu_{q_{2}}+\mu_{\bar{Q}} \\
\frac{1}{2}\left(2 \mu_{1}+\mu_{Q}+\mu_{\mathrm{q} 1}+\mu_{q_{2}}+\mu_{q_{3}}+2 \mu_{\bar{Q}}\right) \\
\frac{1}{10}\left(10 \mu_{1}+9 \mu_{Q}+9 \mu_{q_{1}}+9 \mu_{\mathrm{q} 2}+9 \mu_{q_{3}}-6 \mu_{\bar{Q}}\right)\end{array}$ \\
\hline & ${ }^{6} P_{\frac{5}{2}}$ & $\left(\left(1^{+} \otimes 1^{+}\right)_{2} \otimes \frac{1}{2}^{-}\right)_{\frac{5}{2}} \otimes 1^{-}$ & $\frac{1}{35}\left(10 \mu_{1}+31\left(\mu_{Q}+\mu_{q_{1}}+\mu_{\mathrm{q} 2}+\mu_{q_{3}}+\mu_{\bar{Q}}\right)\right)$ \\
\hline$\frac{7}{2}^{+}$ & ${ }^{6} P_{\frac{5}{2}}$ & $1^{+} \otimes 1^{+} \otimes \frac{1}{2}^{-} \otimes 1^{-}$ & $\mu_{1}+\mu_{Q}+\mu_{q_{1}}+\mu_{\mathrm{q} 2}+\mu_{q_{3}}+\mu_{\bar{Q}}$ \\
\hline
\end{tabular}


TABLE VII: The magnetic moment of the pentaquark states in the diquark-diquark-antiquark model with the configuration $\left(c q_{3}\right)\left(q_{1} q_{2}\right) \bar{c}$ and the isospin $\left(I, I_{3}\right)=\left(\frac{1}{2}, \frac{1}{2}\right)$. They are in the $8_{2 f}$ representation from $\overline{3}_{f} \otimes 3_{f}=1_{f} \oplus 8_{2 f}$ and $8_{1 f}$ representation from $6_{f} \otimes 3_{f}=10_{f} \oplus 8_{1 f}$, respectively. The third line denotes the $J^{P}$ of the diquark $\left(c q_{3}\right)$, diquark $\left(q_{1} q_{2}\right)$, antiquark, and orbital excitation, respectively. For the pentaquark states with positive parity, the $\mathrm{P}$-wave excitation is the $\rho$ mode. The unit is the magnetic moment of the proton.

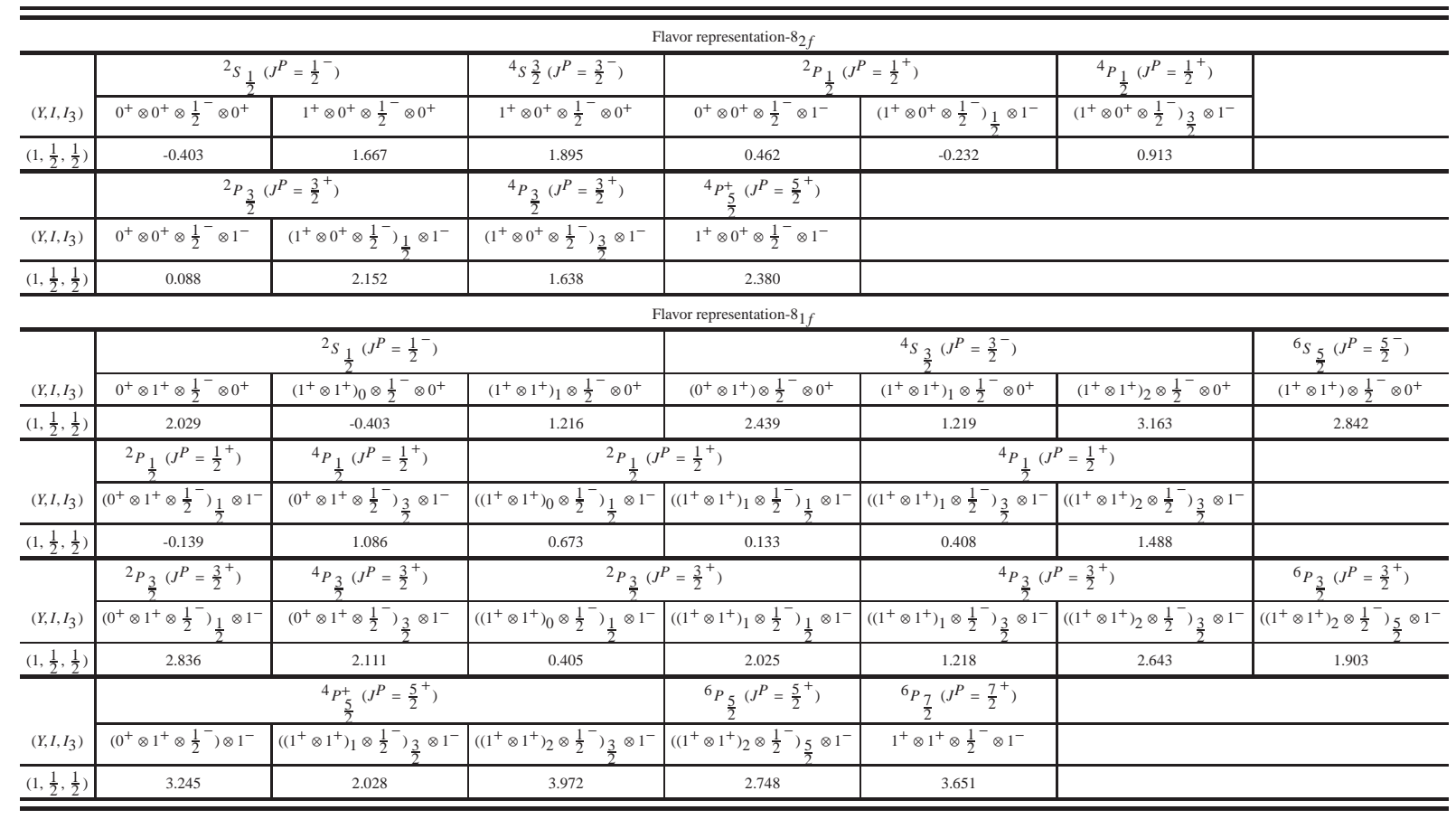

The diquark-triquark model has a similar configuration as the molecular model except the color representation. We also assume that the P-wave excitation exists between the two clusters. The derivation of the magnetic moment in this model is similar to that of the molecule case. Now we have

$$
\begin{aligned}
\vec{\mu} & =g_{d} \mu_{d} \vec{S}_{d}+g_{t} \mu_{t} \vec{S}_{t}+\mu_{l} \vec{l} \\
g_{d} \mu_{d} \vec{S}_{d} & =g_{Q} \mu_{Q} \frac{\overrightarrow{1}}{2}+g_{q_{3}} \mu_{q_{3}} \frac{\overrightarrow{1}}{2} \\
g_{t} \mu_{t} \vec{S}_{t} & =g_{\bar{Q}} \mu_{\bar{Q}} \frac{\overrightarrow{1}}{2}+g_{q_{1}} \mu_{q_{1}} \frac{\overrightarrow{1}}{2}+g_{q_{2}} \mu_{q_{2}} \frac{\overrightarrow{1}}{2} \\
\mu_{l} & =\frac{m_{t} \mu_{d}+m_{d} \mu_{t}}{m_{d}+m_{t}} .
\end{aligned}
$$

The subscripts $d$ and $t$ represent the diquark and triquark, respectively. Similarly,

$$
\begin{gathered}
|J J\rangle=\left|\left[\left[\left(\left(q_{1} q_{2}\right)_{s_{12}} \bar{c}_{\frac{1}{2}}\right)_{S_{t}} \otimes\left(c q_{3}\right)_{S_{d}}\right]_{S} \otimes l\right]_{J}^{J}\right\rangle, \\
\mu=\left\langle J J\left|g_{d} \mu_{d} \vec{S}_{d}+g_{t} \mu_{t} \vec{S}_{t}+\mu_{l} \vec{l}\right| J J\right\rangle \\
=\sum_{m, S_{z}}\left\langle l m, S S_{z} \mid J J\right\rangle^{2}\left\{\mu_{l} m+\sum_{S_{z}^{\prime}}\left\langle S_{t} S_{z}^{\prime}, S_{d}\left(S_{z}-S_{z}^{\prime}\right) \mid S S_{z}\right\rangle^{2}\left[\left(S_{z}-S_{z}^{\prime}\right)\left(\mu_{Q}+\mu_{q_{3}}\right)\right.\right. \\
\left.\left.+\left\langle\frac{1}{2} \frac{1}{2}, s_{12}\left(S_{z}^{\prime}-\frac{1}{2}\right) \mid S_{t} S_{z}^{\prime}\right\rangle^{2}\left(\mu_{\bar{Q}}+\left(S_{z}^{\prime}-\frac{1}{2}\right)\left(\mu_{q_{1}}+\mu_{q_{2}}\right)\right)+\left\langle\frac{1}{2}-\frac{1}{2}, S_{12}\left(S_{z}^{\prime}+\frac{1}{2}\right) \mid S_{t} S_{z}^{\prime}\right\rangle^{2}\left(-\mu_{\bar{Q}}+\left(S_{z}^{\prime}+\frac{1}{2}\right)\left(\mu_{q_{1}}+\mu_{q_{2}}\right)\right)\right]\right\} .
\end{gathered}
$$

where we have replaced the spin of the baryon $S_{b}$ and the spin of the meson $S_{m}$ in Eq. (3) and Eq. (4) by the spin of the diquark $S_{d}$ and the spin of the triquark $S_{t} . m, S_{z}$, and $S_{z}^{\prime}$ are the third component of the orbital excitation, the pentaquark total spin and the triquark spin respectively. $s_{12}$ is the total spin of the diquark inside the triquark. 
TABLE VIII: The magnetic moments of the pentaquark states in the diquark-diquark-antiquark model with the configuration $\left(c q_{3}\right)\left(q_{1} q_{2}\right) \bar{c}$ and the isospin $\left(I, I_{3}\right)=\left(\frac{1}{2}, \frac{1}{2}\right)$. They are in the $8_{2 f}$ representation from $\overline{3}_{f} \otimes 3_{f}=1_{f} \oplus 8_{2 f}$ and $8_{1 f}$ representation from $6_{f} \otimes 3_{f}=10_{f} \oplus 8_{1 f}$ respectively. The third line denotes the $J^{P}$ of the diquark $\left(c q_{3}\right)$, diquark $\left(q_{1} q_{2}\right)$, antiquark and orbital excitation, respectively. For the pentaquark states with positive parity, the $\lambda$ mode $\mathrm{P}$-wave excitation is between the $\bar{c}$ and center of mass system of diquark $\left(c q_{3}\right)$ and diquark $\left(q_{1} q_{2}\right)$. The unit is the magnetic moment of the proton.

\begin{tabular}{|c|c|c|c|c|c|c|c|}
\hline \multicolumn{8}{|c|}{ Flavor representation- $82 f$} \\
\hline & \multicolumn{2}{|c|}{${ }^{2} S_{\frac{1}{2}}\left(J^{P}=\frac{1}{2}^{-}\right)$} & ${ }^{4} S \frac{3}{2}\left(J^{P}=\frac{3}{2}^{-}\right)$ & \multicolumn{2}{|c|}{${ }^{2} P_{\frac{1}{2}}\left(J^{P}=\frac{1}{2}^{+}\right)$} & \multirow{2}{*}{$\begin{array}{c}{ }^{4} P_{\frac{1}{2}}\left(J^{P}=\frac{1}{2}^{+}\right) \\
\left(1^{+} \otimes 0^{+} \otimes \frac{1}{2}^{-}\right) \frac{3}{2} \otimes 1^{-}\end{array}$} & \\
\hline$\left(Y, I, I_{3}\right)$ & $0^{+} \otimes 0^{+} \otimes \frac{1}{2}^{-} \otimes 0^{+}$ & $1^{+} \otimes 0^{+} \otimes \frac{1}{2}^{-} \otimes 0^{+}$ & $1^{+} \otimes 0^{+} \otimes \frac{1}{2}^{-} \otimes 0^{+}$ & $0^{+} \otimes 0^{+} \otimes \frac{1}{2}^{-} \otimes 1^{-}$ & $\left(1^{+} \otimes 0^{+} \otimes \frac{1}{2}^{-}\right)_{\frac{1}{2}} \otimes 1^{-}$ & & \\
\hline \multirow[t]{2}{*}{$\left(1, \frac{1}{2}, \frac{1}{2}\right)$} & -0.403 & 1.667 & 1.895 & 0.675 & 0.854 & 0.348 & \\
\hline & \multicolumn{2}{|c|}{${ }^{2} P_{\frac{3}{2}}\left(J^{P}=\frac{3}{2}^{+}\right)$} & ${ }^{4} P_{\frac{3}{2}}\left(J^{P}=\frac{3}{2}^{+}\right)$ & ${ }^{4} P_{\frac{5}{2}}^{+}\left(J^{P}=\frac{5}{2}^{+}\right)$ & & & \\
\hline$\left(Y, I, I_{3}\right)$ & $0^{+} \otimes 0^{+} \otimes \frac{1}{2}^{-} \otimes 1^{-}$ & $\left(1^{+} \otimes 0^{+} \otimes \frac{1}{2}^{-}\right) \frac{1}{2} \otimes 1^{-}$ & $\left(1^{+} \otimes 0^{+} \otimes \frac{1}{2}^{-}\right) \frac{3}{2} \otimes 1^{-}$ & $1^{+} \otimes 0^{+} \otimes \frac{1}{2}^{-} \otimes 1^{-}$ & & & \\
\hline$\left(1, \frac{1}{2}, \frac{1}{2}\right)$ & 0.408 & 3.781 & 2.235 & 4.009 & & & \\
\hline \multicolumn{8}{|c|}{ Flavor representation- $81 f$} \\
\hline & \multicolumn{3}{|c|}{${ }^{2} S_{\frac{1}{2}}\left(J^{P}=\frac{1}{2}^{-}\right)$} & \multicolumn{3}{|c|}{${ }^{4} S_{\frac{3}{2}}\left(J^{P}=\frac{3^{-}}{2}\right)$} & ${ }^{6} S_{\frac{5}{2}}\left(J^{P}=\frac{5}{2}{ }^{-}\right)$ \\
\hline$\left(Y, I, I_{3}\right)$ & $0^{+} \otimes 1^{+} \otimes \frac{1}{2}^{-} \otimes 0^{+}$ & $\left(1^{+} \otimes 1^{+}\right)_{0} \otimes \frac{1}{2}^{-} \otimes 0^{+}$ & $\left(1^{+} \otimes 1^{+}\right)_{1} \otimes \frac{1}{2}^{-} \otimes 0^{+}$ & $\left(0^{+} \otimes 1^{+}\right) \otimes \frac{1}{2}^{-} \otimes 0^{+}$ & $\left(1^{+} \otimes 1^{+}\right)_{1} \otimes \frac{1}{2}^{-} \otimes 0^{+}$ & $\left(1^{+} \otimes 1^{+}\right)_{2} \otimes \frac{1}{2}^{-} \otimes 0^{+}$ & $\left(1^{+} \otimes 1^{+}\right) \otimes \frac{1}{2}^{-} \otimes 0^{+}$ \\
\hline \multirow[t]{2}{*}{$\left(1, \frac{1}{2}, \frac{1}{2}\right)$} & 2.029 & -0.403 & 1.216 & 2.439 & 1.219 & 3.163 & 2.842 \\
\hline & ${ }^{2} P_{\frac{1}{2}}\left(J^{P}=\frac{1}{2}^{+}\right)$ & ${ }^{4} P_{\frac{1}{2}}\left(J^{P}=\frac{1}{2}^{+}\right)$ & \multicolumn{2}{|c|}{${ }^{2} P_{\frac{1}{2}}\left(J^{P}=\frac{1}{2}^{+}\right)$} & \multicolumn{2}{|c|}{${ }^{4} P_{\frac{1}{2}}\left(J^{P}=\frac{1}{2}^{+}\right)$} & \\
\hline$\left(Y, I, I_{3}\right)$ & $\left(0^{+} \otimes 1^{+} \otimes \frac{1}{2}^{-}\right) \frac{1}{2}^{\otimes 1^{-}}$ & $\left(0^{+} \otimes 1^{+} \otimes \frac{1^{-}}{2}\right)_{\frac{3}{2}} \otimes 1^{-}$ & $\left(\left(1^{+} \otimes 1^{+}\right)_{0} \otimes \frac{1}{2}^{-}\right)_{\frac{1}{2}} \otimes 1^{-}$ & $\left(\left(1^{+} \otimes 1^{+}\right) 1_{1} \frac{1}{2}^{-}\right) \frac{1}{2}{ }^{\otimes 1^{-}}$ & $\left(\left(1^{+} \otimes 1^{+}\right) 1_{1} \otimes \frac{1}{2}^{-}\right) \frac{3}{2} \otimes 1^{-}$ & $\left(\left(1^{+} \otimes 1^{+}\right)_{2} \otimes \frac{1}{2}^{-}\right)_{\frac{3}{2}} \otimes 1^{-}$ & \\
\hline \multirow[t]{2}{*}{$\left(1, \frac{1}{2}, \frac{1}{2}\right)$} & 0.441 & 0.796 & 1.237 & 0.697 & 0.126 & 1.206 & \\
\hline & ${ }^{2} P_{\frac{3}{2}}\left(J^{P}=\frac{3}{2}^{+}\right)$ & ${ }^{4} P_{\frac{3}{2}}\left(J^{P}=\frac{3}{2}^{+}\right)$ & \multicolumn{2}{|c|}{${ }^{2} P_{\frac{3}{2}}\left(J^{P}=\frac{3}{2}^{+}\right)$} & \multicolumn{2}{|c|}{${ }^{4} P_{\frac{3}{2}}\left(J^{P}=\frac{3}{2}^{+}\right)$} & ${ }^{6}{ }^{6} \frac{3}{2}\left(J^{P}=\frac{3}{2}{ }^{+}\right)$ \\
\hline$\left(Y, I, I_{3}\right)$ & $\left(0^{+} \otimes 1^{+} \otimes \frac{1}{2}^{-}\right) \frac{1}{2} \otimes 1^{-}$ & $\left(0^{+} \otimes 1^{+} \otimes \frac{1}{2}^{-}\right)_{\frac{3}{2}} \otimes 1^{-}$ & $\left(\left(1^{+} \otimes 1^{+}\right)_{0} \otimes \frac{1}{2}^{-}\right)_{\frac{1}{2}} \otimes 1^{-}$ & $\left.\left(\left(1^{+} \otimes 1^{+}\right)\right)_{1} \otimes \frac{1}{2}^{-}\right) \frac{1}{2}{ }^{\infty} 1^{-}$ & $\left.\left(\left(1^{+} \otimes 1^{+}\right)\right)_{1} \otimes \frac{1}{2}^{-}\right) \frac{3}{2}{ }^{\otimes 1} 1^{-}$ & $\left(\left(1^{+} \otimes 1^{+}\right)_{2} \otimes \frac{1}{2}^{-}\right)_{\frac{3}{2}} \otimes 1^{-}$ & $\left(\left(1^{+} \otimes 1^{+}\right)_{2} \otimes \frac{1}{2}^{-}\right)_{5} \otimes 1^{-}$ \\
\hline \multirow[t]{3}{*}{$\left(1, \frac{1}{2}, \frac{1}{2}\right)$} & 3.706 & 2.459 & 1.250 & 2.870 & 1.556 & 2.981 & 1.395 \\
\hline & \multicolumn{3}{|c|}{${ }^{4} P_{\frac{5}{2}}^{+}\left(J^{P}=\frac{5}{2}^{+}\right)$} & ${ }^{6} P_{\frac{5}{2}}\left(J^{P}=\frac{5}{2}^{+}\right)$ & ${ }^{6}{ }^{6} \frac{7}{2}\left(J^{P}=\frac{7}{2}^{+}\right)$ & & \\
\hline & $\left(0^{+} \otimes 1^{+} \otimes \frac{1}{2}^{-}\right) \otimes 1^{-}$ & $\left.\left(\left(1^{+} \otimes 1^{+}\right)\right)_{1} \otimes \frac{1}{2}^{-}\right) \frac{3}{2} \otimes 1^{-}$ & $\left(\left(1^{+} \otimes 1^{+}\right) 2 \otimes \frac{1}{2}^{-}\right)_{\frac{3}{2}} \otimes 1^{-}$ & $\left(\left(1^{+} \otimes 1^{+}\right)_{2} \otimes \frac{1}{2}^{-}\right) \frac{5}{2} \otimes^{-}$ & $1^{+} \otimes 1^{+} \otimes \frac{1}{2}^{-} \otimes 1^{-}$ & & \\
\hline$\left(1, \frac{1}{2}, \frac{1}{2}\right)$ & 4.116 & 2.873 & 4.817 & 2.990 & 4.496 & & \\
\hline
\end{tabular}

The masses of the triquark are roughly the sum of the mass of the corresponding diquark and the antiquark. When the $q_{1} q_{2}$ is in the $6_{c}$ representation, their masses are not known. Therefore, we omit results in this case. When the diquark is in the $\overline{3}_{c}$ representation, the numerical results for the pentaquark states with isospin $\frac{1}{2}$ are in Table XI. We also list the analytic formulae of the magnetic moments of the pentaquarks with differen spin and configurations in Table XII. For the pentaquarks with the configuration $\left(q_{1} q_{2}\right)\left(\bar{c} c q_{3}\right)$, we collect the flavor wave functions in Table XIII, and the numerical results for the $\left(I, I_{3}\right)=\left(\frac{1}{2}, \frac{1}{2}\right)$ states are listed in Table XIV.

In Tables XI and XIV, for the states with $J^{P}=\frac{1}{2}^{-}$and configuration $\frac{1}{2}^{-} \otimes 0^{+} \otimes 0^{+}$with the inner structure $\left(c q_{3}\right)\left(q_{1} q_{2} \bar{c}\right)$ or $\left(0 \otimes \frac{1}{2}\right)_{\frac{1}{2}}-\otimes 0^{+} \otimes 0^{+}$with the inner structure $\left(q_{1} q_{2}\right)\left(\bar{c} c q_{3}\right)$, the magnetic moments are the same, which are $-0.403 \mu_{p}$. In Tables VII and VIII, the magnetic moments of the diquark-diquark-antiquark states with $J^{P}=\frac{1}{2}^{-}$and configuration $0^{+} \otimes 0^{+} \otimes \frac{1}{2}^{-} \otimes 0^{+}$are also $-0.403 \mu_{p}$. In this case, the spins of the diquark $\left(c q_{3}\right)$ and diquark $\left(q_{1} q_{2}\right)$ are both 0 in these configurations. Moreover, there is no orbital excitation. Thus, only the anticharm quark contributes to the magnetic moment, which leads to the same negative magnetic moment even for the nine flavor configurations both in the diquark-triquark model and the diquark-diquark-antiquark model.

We collect the numerical results for the states in the $1_{f} \oplus 8_{2 f}$ representation within the diquark-diquark-antiquark scheme in Tables VII and VIII, which can be compared with the results in the diquark-triquark scheme in Tables XI and XIV. In these tables, the magnetic moments of the states without P-wave excitations are the same. However, those of the states with the P-wave excitation are slightly different. In this case, the total spin of $q_{1} q_{2}$ is 0 . The expressions for the magnetic moments are the same within the diquark-triquark and the diquark-diquark-antiquark configurations as illustrated in Tables XII and VI.

The expressions of the magmatic moments of some states in the $8_{2 f} \oplus 10_{f}$ representations are also the same in different models. For example, the magnetic moments of the states with $J^{P}=\frac{1}{2}^{-}$and configuration $\frac{1}{2}^{-} \otimes 0^{+} \otimes 0^{+}$in the diquark-triquark model are the same as those of the states with $J^{P}=\frac{1}{2}^{-}$and configuration $0^{+} \otimes 1^{+} \otimes \frac{1}{2}^{-} \otimes 0^{+}$in the diquark-diquark-antiquark model. For the states with $\mathrm{P}$-wave excitation, their different magnetic moments are due to the different P-wave excitation modes and different masses of the related clusters.

For the molecular-type pentaquark states, their expressions can also be obtained through the interexchange of $\mu_{Q}$ and $\mu_{\bar{Q}}$ in Tables XII. Therefore, the numerical results are usually different. However, for some states without orbital excitation, the 
TABLE IX: The flavor wave functions of the pentaquark states in the diquark-triquark model with the configuration $\left(c q_{3}\right)\left(q_{1} q_{2} \bar{c}\right)$ in different flavor representations from $3_{f} \otimes 3_{f} \otimes 3_{f}=10_{f} \oplus 8_{1 f} \oplus 8_{2 f} \oplus 1_{f}$. Here, $\left\{q_{1} q_{2}\right\}=\frac{1}{\sqrt{2}}\left(q_{1} q_{2}+q_{2} q_{1}\right)$ and $\left[q_{1} q_{2}\right]=\frac{1}{\sqrt{2}}\left(q_{1} q_{2}-q_{2} q_{1}\right)$.

\begin{tabular}{|c|c|c|}
\hline$\left(Y, I, I_{3}\right)$ & Wave function- $8_{1 f}$ & Wave function- $8_{2 f}$ \\
\hline$\left(1, \frac{1}{2}, \frac{1}{2}\right)$ & $-\sqrt{\frac{1}{3}}(c u)(\bar{c}\{u d\})+\sqrt{\frac{2}{3}}(c d)(\bar{c}\{u u\})$ & $(c u)(\bar{c}[u d])$ \\
\hline$\left(1, \frac{1}{2},-\frac{1}{2}\right)$ & $\sqrt{\frac{1}{3}}(c d)(\bar{c}\{u d\})-\sqrt{\frac{2}{3}}(c u)(\bar{c}\{d d\})$ & $(c d)(\bar{c}[u d])$ \\
\hline$\left(-1, \frac{1}{2}, \frac{1}{2}\right)$ & $\sqrt{\frac{1}{3}}(c s)(\bar{c}\{u s\})-\sqrt{\frac{2}{3}}(c u)(\bar{c}\{s s\})$ & $(c s)(\bar{c}[u s])$ \\
\hline$\left(-1, \frac{1}{2},-\frac{1}{2}\right)$ & $\sqrt{\frac{1}{3}}(c s)(\bar{c}\{d s\})-\sqrt{\frac{2}{3}}(c d)(\bar{c}\{s s\})$ & $(c s)(\bar{c}[d s])$ \\
\hline$(0,1,1)$ & $\sqrt{\frac{1}{3}}(c u)(\bar{c}\{u s\})-\sqrt{\frac{2}{3}}(c s)(\bar{c}\{u u\})$ & $(c u)(\bar{c}[u s])$ \\
\hline$(0,1,0)$ & $\sqrt{\frac{1}{6}}[(c d)(\bar{c}\{u s\})+(c u)(\bar{c}\{d s\})]-\sqrt{\frac{2}{3}}(c s)(\bar{c}\{u d\})$ & $\frac{1}{\sqrt{2}}\{(c d)(\bar{c}[u s])+(c u)(\bar{c}[d s])\}$ \\
\hline$(0,1,-1)$ & $\sqrt{\frac{1}{3}}(c d)(\bar{c}\{d s\})-\sqrt{\frac{2}{3}}(c s)(\bar{c}\{d d\})$ & $(c d)(\bar{c}[d s])$ \\
\hline$(0,0,0)$ & $\sqrt{\frac{1}{2}}[(c u)(\bar{c}\{d s\})-(c d)(\bar{c}\{u s\})]$ & $\frac{1}{\sqrt{6}}\{(c d)(\bar{c}[u s])-(c u)(\bar{c}[d s])-2(c s)(\bar{c}[u d])\}$ \\
\hline$\left(Y, I, I_{3}\right)$ & Wave function- $10_{f}$ & Singlet \\
\hline$\left(1, \frac{3}{2}, \frac{3}{2}\right)$ & $(c u)(\bar{c}\{u u\})$ & $\frac{1}{\sqrt{3}}\{(c d)(\bar{c}[u s])-(c u)(\bar{c}[d s])+(c s)(\bar{c}[u d])\}$ \\
\hline$\left(1, \frac{3}{2}, \frac{1}{2}\right)$ & $\sqrt{\frac{2}{3}}(c u)(\bar{c}\{u d\})+\sqrt{\frac{1}{3}}(c d)(\bar{c}\{u u\})$ & \\
\hline$\left(1, \frac{3}{2},-\frac{1}{2}\right)$ & $\sqrt{\frac{2}{3}}(c d)(\bar{c}\{u d\})+\sqrt{\frac{1}{3}}(c u)(\bar{c}\{d d\})$ & \\
\hline$\left(1, \frac{3}{2},-\frac{3}{2}\right)$ & $(c d)(\bar{c}\{d d\})$ & \\
\hline$(0,1,1)$ & $\sqrt{\frac{2}{3}}(c u)(\bar{c}\{u s\})+\sqrt{\frac{1}{3}}(c s)(\bar{c}\{u u\})$ & \\
\hline$(0,1,0)$ & $\sqrt{\frac{1}{3}}[(c d)(\bar{c}\{u s\})+(c u)(\bar{c}\{d s\})]+\sqrt{\frac{1}{3}}(c s)(\bar{c}\{u d\})$ & \\
\hline$(0,1,-1)$ & $\sqrt{\frac{2}{3}}(c d)(\bar{c}\{d s\})+\sqrt{\frac{1}{3}}(c s)(\bar{c}\{d d\})$ & \\
\hline$\left(-1, \frac{1}{2}, \frac{1}{2}\right)$ & $\sqrt{\frac{1}{3}}(c u)(\bar{c}\{s s\})+\sqrt{\frac{2}{3}}(c s)(\bar{c}\{u s\})$ & \\
\hline$\left(-1, \frac{1}{2},-\frac{1}{2}\right)$ & $\sqrt{\frac{1}{3}}(c d)(\bar{c}\{s s\})+\sqrt{\frac{2}{3}}(c s)(\bar{c}\{d s\})$ & \\
\hline$(-2,0,0)$ & $(c s)(\bar{c}\{s s\})$ & \\
\hline
\end{tabular}

TABLE X: Structure of the $6_{c} q_{1} q_{2}$ diquark within the triquark in the diquark-triquark model. Here, S and A represent the wave function of the corresponding space are symmetry and antisymmetry, respectively.

$J_{\text {baryon }}=\frac{1}{2}\left|\begin{array}{ccc}\text { Color } & 6_{c} & \mathrm{~S} \\ \text { Space } & L=0 & \mathrm{~S} \\ \mathrm{Spin} & s=0 & \mathrm{~A} \\ \text { Flavor } & 6_{f} & \mathrm{~S}\end{array}\right| J_{\text {baryon }}=\frac{1}{2}, \frac{3}{2}\left[\begin{array}{ccc}\text { Color } & 6_{c} & \mathrm{~S} \\ \text { Space } & L=0 & \mathrm{~S} \\ \text { Spin } & s=1 & \mathrm{~S} \\ \text { Flavor } & \overline{3}_{f} & \mathrm{~A} \\ \hline\end{array}\right.$

analytic expressions are symmetric under interexchange of $\mu_{Q}$ and $\mu_{\bar{Q}}$ for some special configurations. Then, the magnetic moments of the molecular-type pentaquark states are the same as those of the diquark-triquark-type states with the configuration $\left(c q_{3}\right)\left(\bar{c} q_{1} q_{2}\right)$. For example, the results of the molecular-type states with $J^{P}=\frac{5}{2}^{-}$and configuration $\frac{3}{2}^{+} \otimes 1^{-} \otimes 0^{+}$are the same as those of the diquark-triquark-type states with the configuration $\frac{3}{2}^{-} \otimes 1^{+} \otimes 0^{+}$. Moreover, the magnetic moments of the states with $J^{P}=\frac{5}{2}^{-}$are the same in both the diquark-diquark-antiquark model and diqaurk-triquark model as illustrated in Sec .IV. Thus, their magnetic moments are the same in the above three models. 
TABLE XI: The magnetic moment of the pentaquark states as a diquark-triquark state $\left(c q_{3}\right)\left(q_{1} q_{2} \bar{c}\right)$ with $\left(I, I_{3}\right)=\left(\frac{1}{2}, \frac{1}{2}\right)$. The $8_{1 f}$ and $8_{2 f}$ flavor representations are from the $\overline{3}_{f} \otimes 3_{f}=1_{f} \oplus 8_{2 f}$ and $6_{f} \otimes 3_{f}=10_{f} \oplus 8_{1 f}$, respectively. The wave functions of the pentaquark states are listed in Table IX. The third line denotes the $J^{P}$ of the triquark $q_{1} q_{2} \bar{c}$, the diquark $c q_{3}$, and the orbital excitation. The unit is the magnetic moments of the proton.

\begin{tabular}{|c|c|c|c|c|c|c|c|}
\hline \multicolumn{8}{|c|}{ Flavor representation- $8_{2 f}$} \\
\hline & \multicolumn{2}{|c|}{${ }^{2} S_{\frac{1}{2}}\left(J^{P}=\frac{1}{2}^{-}\right)$} & ${ }^{4} S \frac{3}{2}\left(J^{P}=\frac{3}{2}{ }^{-}\right)$ & \multicolumn{2}{|c|}{${ }^{2} P_{\frac{1}{2}}\left(J^{P}=\frac{1}{2}^{+}\right)$} & ${ }^{4} P_{\frac{1}{2}}\left(J^{P}=\frac{1}{2}{ }^{+}\right)$ & \\
\hline$\left(Y, I, I_{3}\right)$ & $\frac{1}{2}^{-} \otimes 0^{+} \otimes 0^{+}$ & $\frac{1}{2}^{-} \otimes 1^{+} \otimes 0^{+}$ & $\frac{1}{2}^{-} \otimes 1^{+} \otimes 0^{+}$ & $\frac{1}{2}^{-} \otimes 0^{+} \otimes 1^{-}$ & $\left(\frac{1}{2}^{-} \otimes 1^{+}\right)_{\frac{1}{2}} \otimes 1^{-}$ & $\left(\frac{1}{2}^{-} \otimes 1^{+}\right)_{\frac{3}{2}} \otimes 1^{-}$ & \\
\hline \multirow[t]{2}{*}{$\left(1, \frac{1}{2}, \frac{1}{2}\right)$} & -0.403 & 1.667 & 1.895 & 0.317 & -0.384 & 0.967 & \\
\hline & \multicolumn{2}{|c|}{${ }^{2} P_{\frac{3}{2}}\left(J^{P}=\frac{3}{2}^{+}\right)$} & ${ }^{4} P_{\frac{3}{2}}\left(J^{P}=\frac{3}{2}{ }^{+}\right)$ & ${ }^{4} P_{\frac{5}{2}}^{+}\left(J^{P}=\frac{5}{2}^{+}\right)$ & & & \\
\hline$\left(Y, I, I_{3}\right)$ & $\frac{1}{2}^{-} \otimes 0^{+} \otimes 1^{-}$ & $\left(\frac{1}{2}^{-} \otimes 1^{+}\right)_{\frac{1}{2}} \otimes 1^{-}$ & $\left(\frac{1}{2}^{-} \otimes 1^{+}\right)_{\frac{3}{2}} \otimes 1^{-}$ & $\frac{1}{2}^{-} \otimes 1^{+} \otimes 1^{-}$ & & & \\
\hline$\left(1, \frac{1}{2}, \frac{1}{2}\right)$ & -0.129 & 1.924 & 1.493 & 2.153 & & & \\
\hline \multicolumn{8}{|c|}{ Flavor representation- $8_{1 f}$} \\
\hline & \multicolumn{3}{|c|}{${ }^{2} S_{\frac{1}{2}}\left(J^{P}=\frac{1}{2}^{-}\right)$} & \multicolumn{3}{|c|}{${ }^{4} S_{\frac{3}{2}}\left(J^{P}=\frac{3}{2}^{-}\right)$} & ${ }^{6} S_{\frac{5}{2}}^{-}\left(J^{P}=\frac{5}{2}^{-}\right)$ \\
\hline$\left(Y, I, I_{3}\right)$ & $\frac{1}{2}^{-} \otimes 0^{+} \otimes 0^{+}$ & $\frac{1}{2}^{-} \otimes 1^{+} \otimes 0^{+}$ & $\frac{3}{2}^{-} \otimes 1^{+} \otimes 0^{+}$ & $\frac{1}{2}^{-} \otimes 1^{+} \otimes 0^{+}$ & $\frac{3}{2}^{-} \otimes 0^{+} \otimes 0^{+}$ & $\frac{3}{2}^{-} \otimes 1^{+} \otimes 0^{+}$ & $\frac{3}{2}^{-} \otimes 1^{+} \otimes 0^{+}$ \\
\hline \multirow[t]{2}{*}{$\left(1, \frac{1}{2}, \frac{1}{2}\right)$} & 2.029 & -0.408 & 1.221 & 2.433 & 2.439 & 1.950 & 2.842 \\
\hline & \multicolumn{2}{|c|}{${ }^{2} p_{\frac{1}{2}}\left(J^{P}=\frac{1}{2}^{+}\right)$} & \multicolumn{2}{|c|}{${ }^{4} p_{\frac{1}{2}}\left(J^{P}=\frac{1^{+}}{2}\right)$} & ${ }^{2} p_{\frac{1}{2}}\left(J^{P}=\frac{1}{2}^{+}\right)$ & ${ }^{4} p_{\frac{1}{2}}\left(J^{P}=\frac{1}{2}^{+}\right)$ & \\
\hline$\left(Y, I, I_{3}\right)$ & $\frac{1}{2}^{-} \otimes 0^{+} \otimes 1^{-}$ & {$\left[\frac{1}{2}^{-} \otimes 1^{+}\right]_{\frac{1}{2}} \otimes 1^{-}$} & {$\left[\frac{1}{2}^{-} \otimes 1^{+}\right]_{\frac{3}{2}} \otimes 1^{-}$} & $\frac{3}{2}^{-} \otimes 0^{+} \otimes 1^{-}$ & {$\left[\frac{3}{2}^{-} \otimes 1^{+}\right]_{\frac{1}{2}} \otimes 1^{-}$} & {$\left[\frac{3}{2}^{-} \otimes 1^{+}\right]_{\frac{3}{2}} \otimes 1^{-}$} & \\
\hline \multirow[t]{2}{*}{$\left(1, \frac{1}{2}, \frac{1}{2}\right)$} & -0.522 & 0.286 & 1.276 & 1.278 & -0.256 & 1.008 & \\
\hline & \multicolumn{2}{|c|}{${ }^{2} P_{\frac{3}{2}}\left(J^{P}=\frac{3}{2}^{+}\right)$} & \multicolumn{2}{|c|}{${ }^{4} P_{\frac{3}{2}}\left(J^{P}=\frac{3}{2}^{+}\right)$} & ${ }^{2} P_{\frac{3}{2}}\left(J^{P}=\frac{3}{2}^{+}\right)$ & ${ }^{4} P_{\frac{3}{2}}\left(J^{P}=\frac{3}{2}{ }^{+}\right)$ & ${ }^{6} P_{\frac{3}{2}}\left(J^{P}=\frac{3}{2}{ }^{+}\right)$ \\
\hline$\left(Y, I, I_{3}\right)$ & $\frac{1}{2}^{-} \otimes 0^{+} \otimes 1^{-}$ & {$\left[\frac{1}{2}^{-} \otimes 1^{+}\right]_{\frac{1}{2}} \otimes 1^{-}$} & {$\left[\frac{1}{2}^{-} \otimes 1^{+}\right]_{\frac{3}{2}} \otimes 1^{-}$} & $\frac{3}{2}^{-} \otimes 0^{+} \otimes 1^{-}$ & {$\left[\frac{3}{2}^{-} \otimes 1^{+}\right]_{\frac{1}{2}} \otimes 1^{-}$} & {$\left[\frac{3}{2}^{-} \otimes 1^{+}\right]_{\frac{3}{2}} \otimes 1^{-}$} & {$\left[\frac{3}{2}^{-} \otimes 1^{+}\right]_{\frac{5}{2}} \otimes 1^{-}$} \\
\hline \multirow[t]{3}{*}{$\left(1, \frac{1}{2}, \frac{1}{2}\right)$} & 2.262 & -0.182 & 1.874 & 1.882 & 1.446 & 1.520 & 2.252 \\
\hline & \multicolumn{3}{|c|}{${ }^{4} P_{\frac{5}{2}}\left(J^{P}=\frac{5}{2}^{+}\right)$} & ${ }^{6} P_{\frac{5}{2}}\left(J^{P}=\frac{5}{2}^{+}\right)$ & ${ }^{6} P_{\frac{7}{2}}\left(J^{P}=\frac{7}{2}^{+}\right)$ & & \\
\hline & $\frac{1}{2}^{-} \otimes 1^{+} \otimes 1^{-}$ & $\frac{3}{2}^{-} \otimes 0^{+} \otimes 1^{-}$ & {$\left[\frac{3}{2}^{-} \otimes 1^{+}\right]_{\frac{3}{2}} \otimes 1^{-}$} & {$\left[\frac{3}{2}^{-} \otimes 1^{+}\right]_{\frac{5}{2}} \otimes 1^{-}$} & $\frac{3}{2}^{-} \otimes 1^{+} \otimes 1^{-}$ & & \\
\hline$\left(1, \frac{1}{2}, \frac{1}{2}\right)$ & 2.658 & 2.671 & 2.176 & 2.582 & 3.068 & & \\
\hline
\end{tabular}

\section{SUMMARY}

Searching for exotic states is an intriguing issue in hadron physics. In 2015, two candidates of the hidden-charm pentaquark were reported by $\mathrm{LHCb}$ [1], which were confirmed very recently by LHCb by performing a model-independent analysis [2]. The observed $P_{c}(4380)$ and $P_{c}(4450)$ states have stimulated extensive discussions of their inner structures. As summarized in Sec. I, there exist several speculations of the inner structures of $P_{c}(4380)$ and $P_{c}(4450)$, which include the molecular state scheme, diquark-diquark-antiquark scheme and the diquark-triquark scheme. How to distinguish these possible configurations of the $P_{c}$ states becomes a crucial task.

In this work, we propose that the magnetic moments of the hidden-charm pentaquarks can be employed to achieve this aim. We have calculated the magnetic moments of the hidden-charm pentaquark states with $J^{P}=\frac{1}{2}^{ \pm}, \frac{3}{2}^{ \pm}, \frac{5}{2}^{ \pm}$and $\frac{7}{2}^{+}$, where we consider different configurations of the hidden-charm pentaquark states.

In fact, the magnetic moments of the states with the same quantum numbers are usually different in the above three models. We take the state with $J^{P}=\frac{7}{2}^{+}$and the isospin $\left(I, I_{z}\right)=\left(\frac{1}{2}, \frac{1}{2}\right)$ in the $8_{1 f}$ representation as an example. Its magnetic moment is $2.945 \mu_{p}, 3.651 \mu_{p}, 4.496 \mu_{p}, 3.068 \mu_{p}$ and $3.666 \mu_{p}$ when it is a molecular-type state, a diquark-diquark-antiquark-type state with the $\rho$ excitation mode, a diquark-diquark-antiquark-type state with the $\lambda$ excitation mode, a diquark-triquark-type state with the 
TABLE XII: The analytic formula for the magnetic moment of the pentaquark state in the configuration $\left(Q q_{3}\right)\left(\bar{Q} q_{1} q_{2}\right)$ when $s_{q_{1} q_{2}}=0$ or 1 .

\begin{tabular}{|c|c|c|c|}
\hline \multicolumn{4}{|r|}{$s_{q_{1} q_{2}}=0$} \\
\hline$J^{P}$ & ${ }^{2 s+1} L_{J}$ & & Formula \\
\hline$\frac{1}{2}^{-}$ & ${ }^{2} S_{\frac{1}{2}}$ & $\begin{array}{l}\frac{1}{2}^{-} \otimes 0^{+} \otimes 0^{+} \\
\frac{1}{2}^{-} \otimes 1^{+} \otimes 0^{+}\end{array}$ & $\begin{array}{c}\mu_{\bar{Q}} \\
\frac{1}{3}\left(2 \mu_{Q}+2 \mu_{q_{3}}-\mu_{\bar{Q}}\right) \\
\end{array}$ \\
\hline$\frac{3}{2}^{-}$ & ${ }^{4} S_{\frac{3}{2}}$ & $\frac{1}{2}^{-} \otimes 1^{+} \otimes 0^{+}$ & $\mu_{Q}+\mu_{q_{3}}+\mu_{\bar{Q}}$ \\
\hline \multirow[t]{2}{*}{$\frac{1}{2}^{+}$} & ${ }^{2} P_{\frac{1}{2}}$ & $\begin{array}{c}\frac{1}{2}^{-} \otimes 0^{+} \otimes 1^{-} \\
\left(\frac{1}{2}^{-} \otimes 1^{+}\right)_{\frac{1}{2}} \otimes 1^{-}\end{array}$ & $\begin{array}{c}\frac{1}{3}\left(2 \mu_{1}-\mu_{\bar{Q}}\right) \\
\frac{1}{9}\left(6 \mu_{1}-2 \mu_{Q}-2 \mu_{q_{3}}+\mu_{\bar{Q}}\right)\end{array}$ \\
\hline & ${ }^{4} P_{\frac{1}{2}}$ & $\left(\frac{1}{2}^{-} \otimes 1^{+}\right)_{\frac{3}{2}} \otimes 1^{-}$ & $\frac{1}{9}\left(-3 \mu_{1}+5\left(\mu_{Q}+\mu_{q_{3}}+\mu_{\bar{Q}}\right)\right.$ \\
\hline \multirow[t]{2}{*}{$\frac{3}{2}^{+}$} & ${ }^{2} P_{\frac{3}{2}}$ & $\begin{array}{c}\frac{1}{2}^{-} \otimes 0^{+} \otimes 1^{-} \\
\left(\frac{1}{2}^{-} \otimes 1^{+}\right)_{\frac{1}{2}} \otimes 1^{-}\end{array}$ & $\begin{array}{c}\mu_{1}+\mu_{\bar{Q}} \\
\frac{1}{3}\left(3 \mu_{1}+2 \mu_{Q}+2 \mu_{q_{3}}-\mu_{\bar{Q}}\right) \\
\end{array}$ \\
\hline & ${ }^{4} P_{\frac{3}{2}}$ & $\left(\frac{1}{2}^{-} \otimes 1^{+}\right)_{\frac{3}{2}} \otimes 1^{-}$ & $\frac{1}{15}\left(6 \mu_{1}+11\left(\mu_{Q}+\mu_{q_{3}}+\mu_{\bar{Q}}\right)\right)$ \\
\hline$\frac{5}{2}^{+}$ & ${ }^{4} P_{\frac{5}{2}}$ & $\frac{1}{2}^{-} \otimes 1^{+} \otimes 1^{-}$ & $\mu_{1}+\mu_{Q}+\mu_{q_{3}}+\mu_{\bar{Q}}$ \\
\hline \multicolumn{4}{|r|}{$s_{q_{1} q_{2}}=1$} \\
\hline$J^{P}$ & ${ }^{2 s+1} L_{J}$ & & Formula \\
\hline$\frac{1}{2}^{-}$ & ${ }^{2} S_{\frac{1}{2}}$ & $\begin{array}{l}\frac{1}{2}^{-} \otimes 0^{+} \otimes 0^{+} \\
\frac{1}{2}^{-} \otimes 1^{+} \otimes 0^{+} \\
\frac{3}{2}^{-} \otimes 1^{+} \otimes 0^{+}\end{array}$ & $\begin{array}{c}\frac{1}{3}\left(2 \mu_{q_{1}}+2 \mu_{q_{2}}-\mu_{\bar{Q}}\right) \\
\frac{1}{9}\left(6 \mu_{Q}-2 \mu_{q_{1}}-2 \mu_{q_{2}}+6 \mu_{q_{3}}+\mu_{\bar{Q}}\right) \\
\frac{1}{9}\left(-3 \mu_{Q}+5 \mu_{q_{1}}+5 \mu_{q_{2}}-3 \mu_{q_{3}}+5 \mu_{\bar{Q}}\right)\end{array}$ \\
\hline$\frac{3}{2}$ & ${ }^{4} S_{\frac{3}{2}}$ & $\begin{array}{l}\frac{1}{2}^{-} \otimes 0^{+} \otimes 0^{+} \\
\frac{3}{2}^{-} \otimes 0^{+} \otimes 0^{+} \\
\frac{3}{2}^{-} \otimes 1^{+} \otimes 0^{+}\end{array}$ & $\begin{array}{c}\mu_{Q}+\frac{2 \mu_{q_{1}}}{3}+\frac{2 \mu_{q_{2}}}{3}+\mu_{q_{3}}-\frac{\mu_{\bar{Q}}}{3} \\
\mu_{q_{1}}+\mu_{q_{2}}+\mu_{\bar{Q}} \\
\frac{1}{15}\left(6 \mu_{Q}+11 \mu_{q_{1}}+11 \mu_{q_{2}}+6 \mu_{q_{3}}+11 \mu_{\bar{Q}}\right)\end{array}$ \\
\hline$\frac{5}{2}^{-}$ & ${ }^{6} S_{\frac{5}{2}}$ & $\frac{3}{2}^{-} \otimes 1^{+} \otimes 0^{+}$ & $\mu_{Q}+\mu_{q_{1}}+\mu_{q_{2}}+\mu_{q_{3}}+\mu_{\bar{Q}}$ \\
\hline \multirow[t]{3}{*}{$\frac{1}{2}^{+}$} & ${ }^{2} P_{\frac{1}{2}}$ & $\begin{array}{c}\frac{1}{2}^{-} \otimes 0^{+} \otimes 1^{-} \\
\left.\frac{1}{2}^{-} \otimes 1^{+}\right]_{\frac{1}{2}} \otimes 1^{-}\end{array}$ & $\begin{array}{c}\frac{1}{9}\left(6 \mu_{1}-2 \mu_{q_{1}}-2 \mu_{q_{2}}+\mu_{\bar{Q}}\right) \\
\frac{1}{27}\left(18 \mu_{1}-6 \mu_{Q}+2 \mu_{q_{1}}+2 \mu_{q_{2}}-6 \mu_{q_{3}}-\mu_{\bar{Q}}\right) \\
\end{array}$ \\
\hline & ${ }^{4} P_{\frac{1}{2}}$ & 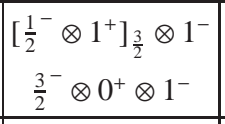 & $\begin{array}{c}\frac{1}{27}\left(-9 \mu_{1}+5\left(3 \mu_{Q}+2 \mu_{q_{1}}+2 \mu_{q_{2}}+3 \mu_{q_{3}}-\mu_{\bar{Q}}\right)\right) \\
\frac{1}{9}\left(-3 \mu_{1}+5\left(\mu_{q_{1}}+\mu_{q_{2}}+\mu_{\bar{Q}}\right)\right) \\
\end{array}$ \\
\hline & ${ }^{2} P_{\frac{1}{2}}$ & $\begin{array}{l}{\left[\frac{3}{2}^{-} \otimes 1^{+}\right]_{\frac{1}{2}} \otimes 1^{-}} \\
{\left[\frac{3}{2}^{-} \otimes 1^{+}\right]_{\frac{3}{2}} \otimes 1^{-}}\end{array}$ & $\begin{array}{c}\frac{1}{27}\left(18 \mu_{1}+3 \mu_{Q}-5 \mu_{q_{1}}-5 \mu_{q_{2}}+3 \mu_{q_{3}}-5 \mu_{\bar{Q}}\right) \\
\frac{1}{27}\left(-9 \mu_{1}+6 \mu_{Q}+11 \mu_{q_{1}}+11 \mu_{q_{2}}+6 \mu_{q_{3}}+11 \mu_{\bar{Q}}\right)\end{array}$ \\
\hline \multirow[t]{3}{*}{$\frac{3}{2}^{+}$} & ${ }^{2} P_{\frac{3}{2}}$ & $\begin{array}{c}\frac{1}{2}^{-} \otimes 0^{+} \otimes 1^{-} \\
\left.\frac{1}{2}^{-} \otimes 1^{+}\right]_{\frac{1}{2}} \otimes 1^{-}\end{array}$ & $\begin{array}{c}\frac{1}{3}\left(3 \mu_{1}+2 \mu_{q_{1}}+2 \mu_{q_{2}}-\mu_{\bar{Q}}\right) \\
\frac{1}{9}\left(9 \mu_{1}+6 \mu_{Q}-2 \mu_{q_{1}}-2 \mu_{q_{2}}+6 \mu_{q_{3}}+\mu_{\bar{Q}}\right)\end{array}$ \\
\hline & ${ }^{4} P_{\frac{3}{2}}$ & $\begin{array}{c}\left.{\frac{1^{2}}{}}^{-} \otimes 1^{+}\right]_{\frac{3}{2}} \otimes 1^{-} \\
\frac{3}{2}^{-} \otimes 0^{+} \otimes 1^{-}\end{array}$ & $\begin{array}{c}\frac{1}{45}\left(18 \mu_{1}+11\left(3 \mu_{Q}+2 \mu_{q_{1}}+2 \mu_{q_{2}}+3 \mu_{q_{3}}-\mu_{\bar{Q}}\right)\right) \\
\frac{1}{15}\left(6 \mu_{1}+11\left(\mu_{q_{1}}+\mu_{q_{2}}+\mu_{\bar{Q}}\right)\right) \\
\end{array}$ \\
\hline & $\begin{array}{l}{ }^{2} P_{\frac{3}{2}} \\
{ }^{4} P_{\frac{3}{2}} \\
{ }^{6} P_{\frac{3}{2}} \\
\end{array}$ & $\begin{array}{l}{\left[\frac{3}{2}^{-} \otimes 1^{+}\right]_{\frac{1}{2}} \otimes 1^{-}} \\
{\left[\frac{3}{2}^{-} \otimes 1^{+}\right]_{\frac{3}{2}} \otimes 1^{-}} \\
{\left[\frac{3}{2}^{-} \otimes 1^{+}\right]_{\frac{5}{2}} \otimes 1^{-}}\end{array}$ & $\begin{array}{c}\frac{1}{9}\left(9 \mu_{1}-3 \mu_{Q}+5 \mu_{q_{1}}+5 \mu_{q_{2}}-3 \mu_{q_{3}}+5 \mu_{\bar{Q}}\right) \\
\frac{1}{225}\left(90 \mu_{1}+11\left(6 \mu_{Q}+11 \mu_{q_{1}}+11 \mu_{q_{2}}+6 \mu_{q_{3}}+11 \mu_{\bar{Q}}\right)\right) \\
\frac{3}{25}\left(-5 \mu_{1}+7\left(\mu_{Q}+\mu_{q_{1}}+\mu_{q_{2}}+\mu_{q_{3}}+\mu_{\bar{Q}}\right)\right) \\
\end{array}$ \\
\hline \multirow[t]{2}{*}{$\frac{5}{2}^{+}$} & ${ }^{4} P_{\frac{5}{2}}$ & $\mid \begin{array}{c}\frac{1}{2}^{-} \otimes 1^{+} \otimes 1^{-} \\
\frac{3}{2}^{-} \otimes 0^{+} \otimes 1^{-} \\
\left.\frac{3}{2}^{-} \otimes 1^{+}\right]_{\frac{3}{2}} \otimes 1^{-}\end{array}$ & $\begin{array}{c}\mu_{1}+\mu_{Q}+\frac{2 \mu_{q_{1}}}{3}+\frac{2 \mu_{q_{2}}}{3}+\mu_{q_{3}}-\frac{\mu_{\bar{Q}}}{3} \\
\mu_{1}+\mu_{q_{1}}+\mu_{q_{2}}+\mu_{\bar{Q}} \\
\frac{1}{15}\left(15 \mu_{1}+6 \mu_{Q}+11 \mu_{q_{1}}+11 \mu_{q_{2}}+6 \mu_{q_{3}}+11 \mu_{\bar{Q}}\right)\end{array}$ \\
\hline & ${ }^{6} P_{\frac{5}{2}}$ & {$\left[\frac{3}{2}^{-} \otimes 1^{+}\right]_{\frac{5}{2}} \otimes 1^{-}$} & $\frac{1}{35}\left(10 \mu_{1}+31\left(\mu_{Q}+\mu_{q_{1}}+\mu_{q_{2}}+\mu_{q_{3}}+\mu_{\bar{Q}}\right)\right)$ \\
\hline$\frac{7}{2}^{+}$ & ${ }^{6} P_{\frac{7}{2}}$ & $\frac{3}{2}^{-} \otimes 1^{+} \otimes 1^{-}$ & $\mu_{1}+\mu_{Q}+\mu_{q_{1}}+\mu_{q_{2}}+\mu_{q_{3}}+\mu_{\bar{Q}}$ \\
\hline
\end{tabular}


TABLE XIII: The flavor wave functions of the pentaquark states in the diquark-triquark model with the configuration $\left(q_{1} q_{2}\right)\left(\bar{c} c q_{3}\right)$ in the different flavor representation from $3_{f} \otimes 3_{f} \otimes 3_{f}=10_{f} \oplus 8_{1 f} \oplus 8_{2 f} \oplus 1_{f}$. Here, $\left\{q_{1} q_{2}\right\}=\frac{1}{\sqrt{2}}\left(q_{1} q_{2}+q_{2} q_{1}\right),\left[q_{1} q_{2}\right]=\frac{1}{\sqrt{2}}\left(q_{1} q_{2}-q_{2} q_{1}\right)$.

\begin{tabular}{|c|c|c|}
\hline$\left(Y, I, I_{3}\right)$ & Wave function- $8_{1 f}$ & Wave function- $8_{2 f}$ \\
\hline$\left(1, \frac{1}{2}, \frac{1}{2}\right)$ & $-\sqrt{\frac{1}{3}}\{u d\}(\bar{c} c u)+\sqrt{\frac{2}{3}}\{u u\}(\bar{c} c d)$ & {$[u d](\bar{c} c u)$} \\
\hline$\left(1, \frac{1}{2},-\frac{1}{2}\right)$ & $\sqrt{\frac{1}{3}}\{u d\}(\bar{c} c d)-\sqrt{\frac{2}{3}}\{d d\}(\bar{c} c u)$ & {$[u d](\bar{c} c d)$} \\
\hline$\left(-1, \frac{1}{2}, \frac{1}{2}\right)$ & $\sqrt{\frac{1}{3}}\{u s\}(\bar{c} c s)-\sqrt{\frac{2}{3}}\{s s\}(\bar{c} c u)$ & {$[u s](\bar{c} c s)$} \\
\hline$\left(-1, \frac{1}{2},-\frac{1}{2}\right)$ & $\sqrt{\frac{1}{3}}\{d s\}(\bar{c} c s)-\sqrt{\frac{2}{3}}\{s s\}(\bar{c} c d)$ & {$[d s](\bar{c} c s)$} \\
\hline$(0,1,1)$ & $\sqrt{\frac{1}{3}}\{u s\}(\bar{c} c u)-\sqrt{\frac{2}{3}}\{u u\}(\bar{c} c s)$ & {$[u s](\bar{c} c u)$} \\
\hline$(0,1,0)$ & $\sqrt{\frac{1}{6}}[\{u s\}(\bar{c} c d)+\{d s\}(\bar{c} c u)]-\sqrt{\frac{2}{3}}\{u d\}(\bar{c} c s)$ & $\frac{1}{\sqrt{2}}\{[u s](\bar{c} c d)+[d s](\bar{c}) c u\}$ \\
\hline$(0,1,-1)$ & $\sqrt{\frac{1}{3}}\{d s\}(\bar{c} c d)-\sqrt{\frac{2}{3}}\{d d\}(\bar{c} c s)$ & {$[d s](\bar{c} c d)$} \\
\hline$(0,0,0)$ & $\sqrt{\frac{1}{2}}[\{d s\}(\bar{c} c u)-(\{u s\})(\bar{c} c d)]$ & $\frac{1}{\sqrt{6}}\{[u s](\bar{c} c d)-[d s](\bar{c} c u)-2[u d](\bar{c} c s)\}$ \\
\hline$\left(Y, I, I_{3}\right)$ & Wave function- $10_{f}$ & Singlet \\
\hline$\left(1, \frac{3}{2}, \frac{3}{2}\right)$ & $\{u u\}(\bar{c} c u)$ & $\frac{1}{\sqrt{3}}\{[u s](\bar{c} c d)-[d s](\bar{c} c u)+[u d](\bar{c} c s)\}$ \\
\hline$\left(1, \frac{3}{2}, \frac{1}{2}\right)$ & $\sqrt{\frac{2}{3}}\{u d\}(\bar{c} c u)+\sqrt{\frac{1}{3}}\{u u\}(\bar{c} c d)$ & \\
\hline$\left(1, \frac{3}{2},-\frac{1}{2}\right)$ & $\sqrt{\frac{2}{3}}\{u d\}(\bar{c} c d)+\sqrt{\frac{1}{3}}\{d d\}(\bar{c} c u)$ & \\
\hline$\left(1, \frac{3}{2},-\frac{3}{2}\right)$ & $\{d d\}(\bar{c} c d)$ & \\
\hline$(0,1,1)$ & $\sqrt{\frac{2}{3}}\{u s\}(\bar{c} c u)+\sqrt{\frac{1}{3}}\{u u\}(\bar{c} c s)$ & \\
\hline$(0,1,0)$ & $\sqrt{\frac{1}{3}}[\{u s\}(\bar{c} c d)+\{d s\}(\bar{c} c u)]+\sqrt{\frac{1}{3}}\{u d\}(\bar{c} c s)$ & \\
\hline$(0,1,-1)$ & $\sqrt{\frac{2}{3}}\{d s\}(\bar{c} c d)+\sqrt{\frac{1}{3}}\{d d\}(\bar{c} c s)$ & \\
\hline$\left(-1, \frac{1}{2}, \frac{1}{2}\right)$ & $\sqrt{\frac{1}{3}}\{s s\}(\bar{c} c u)+\sqrt{\frac{2}{3}}\{u s\}(\bar{c} c s)$ & \\
\hline$\left(-1, \frac{1}{2},-\frac{1}{2}\right)$ & $\sqrt{\frac{1}{3}}\{s s\}(\bar{c} d)+\sqrt{\frac{2}{3}}\{d s\}(\bar{c} c s)$ & \\
\hline$(-2,0,0)$ & $\{s s\}(\bar{c} c s)$ & \\
\hline
\end{tabular}

configurations $\left(c q_{3}\right)\left(\bar{c} q_{1} q_{2}\right)$ and $\left(q_{1} q_{2}\right)\left(\bar{c} c q_{3}\right)$, respectively.

Even within the same model, one set of the $J^{P}$ quantum numbers may correspond to several states with different configurations. Their magnetic moments are also different. Let's take the magnetic moments of the hidden charm molecular states composed of $\Sigma_{c} \bar{D}^{*}$ in the $8_{1 f}$ representation with $J^{P}=\frac{3}{2}^{+}$and the isospin $\left(I, I_{z}\right)=\left(\frac{1}{2}, \frac{1}{2}\right)$ in Table IV as an example. The magnetic moments of the pentaquark states with different configurations $\left[\frac{1}{2}^{+} \otimes 1^{-}\right]_{\frac{1}{2}} \otimes 1^{-}$and $\left[\frac{1}{2}^{+} \otimes 1^{-}\right]_{\frac{3}{2}} \otimes 1^{-}$are very different, which are $-0.740 \mu_{p}$ and $1.041 \mu_{p}$, respectively.

In the diquark-diquark-antiquark model, we choose the states with the isospin $\left(I, I_{z}\right)=\left(\frac{1}{2}, \frac{1}{2}\right)$ in the $8_{1 f}$ representation as an example. In Tables VII and VIII, the magnetic moments of the $J^{P}=\frac{3}{2}^{+}$states with the p-wave $\rho$ excitation mode and configurations $\left(\left(1^{+} \otimes 1^{+}\right)_{0} \otimes \frac{1}{2}^{-}\right)_{\frac{1}{2}} \otimes 1^{-}$and $\left(\left(1^{+} \otimes 1^{+}\right)_{1} \otimes \frac{1}{2}^{-}\right)_{\frac{1}{2}} \otimes 1^{-}$are $0.405 \mu_{p}$ and $2.025 \mu_{p}$, respectively, while the corresponding numerical results of these two configurations with the $\lambda$ excitation mode are $1.250 \mu_{p}$ and $2.870 \mu_{p}$, respectively. The results of the states with the same p-wave excitation mode are different if their configurations are different. Moreover, the different p-wave excitation modes also lead to the different numerical results. Actually, the magnetic moments of the $\frac{3}{2}^{+}$and $\frac{5}{2}^{+} P_{c}$ states in the $8_{1 f}$ representation with the isospin $\left(I, I_{z}\right)=\left(\frac{1}{2}, \frac{1}{2}\right)$ and the $\lambda$ excitation mode are larger than those of the states with the $\rho$ excitation mode except the configuration $\left(\left(1^{+} \otimes 1^{+}\right)_{2} \otimes \frac{1}{2}^{-}\right)_{\frac{5}{2}} \otimes 1^{-}$as illustrated in Sec. IV.

This also happens in the diquark-triquark model. For example, in Table XI, the magnetic moments of the $\frac{1}{2}^{+}$states as a $\left(c q_{3}\right)\left(q_{1} q_{2} \bar{c}\right)$ state in the $82 f$ flavor representation with configurations $\left(\frac{1}{2}^{-} \otimes 1^{+}\right)_{\frac{1}{2}} \otimes 1^{-}$and $\left(\frac{1}{2}^{-} \otimes 1^{+}\right)_{\frac{3}{2}} \otimes 1^{-}$are $-0.384 \mu_{p}$ and 
TABLE XIV: The magnetic moment of the pentaquark states as a diquark-triquark state $\left(q_{1} q_{2}\right)\left(\bar{c} c q_{3}\right)$ with $\left(I, I_{3}\right)=\left(\frac{1}{2}, \frac{1}{2}\right)$. The $8_{1 f}$ and $8_{2 f}$ flavor representations are from the the $\overline{3}_{f} \otimes 3_{f}=1_{f} \oplus 8_{2 f}$ and $6_{f} \otimes 3_{f}=10_{f} \oplus 8_{1 f}$, respectively. The third line denotes the $J^{P}$ of the diquark $c q_{3}$, the antiquark $\bar{c}$, the diquark $q_{1} q_{2}$, and the orbital excitation. The subscripts $\frac{1}{2}^{-}\left(\frac{3}{2}^{-}\right)$represent the $J^{P}$ of the triquark $\bar{c} c q_{3}$, while the subscripts 0 (or 1) are the total spin of the diquark $c q_{3}$ in the triquark $\bar{c} c q_{3}$. The unit is the magnetic moment of the proton.

\begin{tabular}{|c|c|c|c|c|c|c|c|}
\hline \multicolumn{8}{|c|}{ Flavor representation- $8_{2 f}$} \\
\hline & \multicolumn{2}{|c|}{${ }^{2} S_{\frac{1}{2}}\left(J^{P}=\frac{1}{2}^{-}\right)$} & \multirow{2}{*}{\begin{tabular}{c|}
${ }^{4} S \frac{3}{2}\left(J^{P}=\frac{3}{2}^{-}\right)$ \\
$\left(1 \otimes \frac{1}{2}\right)_{\frac{3}{2}}-\otimes 0^{+} \otimes 0^{+}$ \\
\end{tabular}} & \multicolumn{2}{|c|}{${ }^{2} P_{\frac{1}{2}}\left(J^{P}=\frac{1}{2}^{+}\right)$} & \multirow{2}{*}{$\begin{array}{c}{ }^{4} P_{\frac{1}{2}}\left(J^{P}=\frac{1}{2}^{+}\right) \\
\left(1 \otimes \frac{1}{2}^{-}\right)_{\frac{3}{2}}-\otimes 0^{+} \otimes 1^{-} \\
\end{array}$} & \\
\hline$\left(Y, I, I_{3}\right)$ & $\left(0 \otimes \frac{1}{2}\right) \frac{1}{2}^{-} \otimes 0^{+} \otimes 0^{+}$ & $\left(1 \otimes \frac{1}{2}^{2}\right)_{\frac{1}{2}}-\otimes 0^{+} \otimes 0^{+}$ & & $\left(0 \otimes \frac{1}{2}^{2}\right)_{\frac{1}{2}}-\otimes 0^{+} \otimes 1^{-}$ & $\left(1 \otimes \frac{1}{2}\right)_{\frac{1}{2}}-\otimes 0^{+} \otimes 1^{-}$ & & \\
\hline \multirow[t]{2}{*}{$\left(1, \frac{1}{2}, \frac{1}{2}\right)$} & -0.403 & 1.667 & 1.895 & 0.399 & -0.291 & 0.921 & \\
\hline & \multicolumn{2}{|c|}{${ }^{2} P_{\frac{3}{2}}\left(J^{P}=\frac{3}{2}{ }^{+}\right)$} & ${ }^{4} P_{\frac{3}{2}}\left(J^{P}=\frac{3}{2}^{+}\right)$ & ${ }^{4} P_{\frac{5}{2}}^{+}\left(J^{P}=\frac{5}{2}^{+}\right)$ & & & \\
\hline$\left(Y, I, I_{3}\right)$ & $\left(0 \otimes \frac{1}{2}\right)_{\frac{1}{2}}-\otimes 0^{+} \otimes 1^{-}$ & $\left(1 \otimes \frac{1}{2}\right)_{\frac{1}{2}}-\otimes 0^{+} \otimes 1^{-}$ & $\left(1 \otimes \frac{1}{2}\right)_{\frac{3}{2}}-\otimes 0^{+} \otimes 1^{-}$ & $\left(1 \otimes \frac{1}{2}\right)_{\frac{3}{2}}-\otimes 0^{+} \otimes 1^{-}$ & & & \\
\hline$\left(1, \frac{1}{2}, \frac{1}{2}\right)$ & -0.007 & 2.063 & 1.548 & 2.291 & & & \\
\hline \multicolumn{8}{|c|}{ Flavor representation- $8_{1 f}$} \\
\hline & \multicolumn{3}{|c|}{${ }^{2} S_{\frac{1}{2}}\left(J^{P}=\frac{1}{2}^{-}\right)$} & \multicolumn{3}{|c|}{${ }^{4} S_{\frac{3}{2}}\left(J^{P}=\frac{3}{2}^{-}\right)$} & ${ }^{6} S_{\frac{5}{2}}\left(J^{P}=\frac{5}{2}^{-}\right)$ \\
\hline$\left(Y, I, I_{3}\right)$ & $\frac{1}{2} 0_{0}^{-} \otimes 1^{+} \otimes 0^{+}$ & $\frac{1_{2}^{-}}{1} \otimes 1^{+} \otimes 0^{+}$ & $\frac{3}{2} 1_{1}^{-} \otimes 1^{+} \otimes 0^{+}$ & $\frac{1^{-}}{2} \otimes 1^{+} \otimes 0^{+}$ & $\frac{1_{2}^{-}}{1} \otimes 0^{+} \otimes 0^{+}$ & $\frac{3}{2} 1_{1}^{-} \otimes 1^{+} \otimes 0^{+}$ & $\frac{3}{2} 1_{1}^{-} \otimes 1^{+} \otimes 0^{+}$ \\
\hline \multirow[t]{2}{*}{$\left(1, \frac{1}{2}, \frac{1}{2}\right)$} & 2.029 & 1.760 & -0.947 & 2.439 & 3.246 & 1.137 & 2.842 \\
\hline & ${ }^{2} P_{\frac{1}{2}}\left(J^{P}=\frac{1}{2}^{+}\right)$ & ${ }^{4} P_{\frac{1}{2}}\left(J^{P}=\frac{1}{2}^{+}\right)$ & ${ }^{2} P_{\frac{1}{2}}\left(J^{P}=\frac{1}{2}^{+}\right)$ & ${ }^{4} P_{\frac{1}{2}}\left(J^{P}=\frac{1}{2}^{+}\right)$ & ${ }^{2} P_{\frac{1}{2}}\left(J^{P}=\frac{1}{2}^{+}\right)$ & ${ }^{4} P_{\frac{1}{2}}\left(J^{P}=\frac{1}{2}^{+}\right)$ & \\
\hline$\left(Y, I, I_{3}\right)$ & {$\left[\frac{1}{2}_{0}^{-} \otimes 1^{+}\right]_{\frac{1}{2}} \otimes 1^{-}$} & {$\left[\frac{1}{2}_{0}^{-} \otimes 1^{+}\right]_{\frac{3}{2}} \otimes 1^{-}$} & {$\left[\frac{1}{2}_{1}^{-} \otimes 1^{+}\right]_{\frac{1}{2}} \otimes 1^{-}$} & {$\left[\frac{1}{2}_{1}^{-} \otimes 1^{+}\right]_{\frac{3}{2}} \otimes 1^{-}$} & {$\left[\frac{3}{2}_{1}^{-} \otimes 1^{+}\right]_{\frac{1}{2}} \otimes 1^{-}$} & {$\left[\frac{3}{2}_{1}^{-} \otimes 1^{+}\right]_{\frac{3}{2}} \otimes 1^{-}$} & \\
\hline \multirow[t]{2}{*}{$\left(1, \frac{1}{2}, \frac{1}{2}\right)$} & -0.130 & 1.082 & -0.038 & 1.529 & 0.865 & 0.357 & \\
\hline & ${ }^{2} P_{\frac{3}{2}}\left(J^{P}=\frac{3}{2}^{+}\right)$ & ${ }^{4} P_{\frac{3}{2}}\left(J^{P}=\frac{3}{2}^{+}\right)$ & ${ }^{2} P_{\frac{3}{2}}\left(J^{P}=\frac{3}{2}^{+}\right)$ & ${ }^{4} P_{\frac{3}{2}}\left(J^{P}=\frac{3}{2}^{+}\right)$ & ${ }^{2} P_{\frac{3}{2}}\left(J^{P}=\frac{3}{2}^{+}\right)$ & ${ }^{4} P_{\frac{3}{2}}\left(J^{P}=\frac{3}{2}^{+}\right)$ & ${ }^{6} P_{\frac{3}{2}}\left(J^{P}=\frac{3}{2}{ }^{+}\right)$ \\
\hline$\left(Y, I, I_{3}\right)$ & {$\left[\frac{1}{2}_{0}^{-} \otimes 1^{+}\right]_{\frac{1}{2}} \otimes 1^{-}$} & {$\left[\frac{1}{2}_{0}^{-} \otimes 1^{+}\right]_{\frac{3}{2}} \otimes 1^{-}$} & {$\left[\frac{1}{2}_{1}^{-} \otimes 1^{+}\right]_{\frac{1}{2}} \otimes 1^{-}$} & {$\left[\frac{1}{2}_{1}^{-} \otimes 1^{+}\right]_{\frac{3}{2}} \otimes 1^{-}$} & {$\left[\frac{3}{2}_{1}^{-} \otimes 1^{+}\right]_{\frac{1}{2}} \otimes 1^{-}$} & {$\left[\frac{3}{2}_{1}^{-} \otimes 1^{+}\right]_{\frac{3}{2}} \otimes 1^{-}$} & {$\left[\frac{3}{2}_{1}^{-} \otimes 1^{+}\right]_{\frac{5}{2}} \otimes 1^{-}$} \\
\hline \multirow[t]{2}{*}{$\left(1, \frac{1}{2}, \frac{1}{2}\right)$} & 2.850 & 2.117 & 2.584 & 2.710 & -0.124 & 1.163 & 1.894 \\
\hline & \multicolumn{3}{|c|}{${ }^{4} P_{\frac{5}{2}}\left(J^{P}=\frac{5}{2}^{+}\right)$} & ${ }^{6} P_{\frac{5}{2}}\left(J^{P}=\frac{5}{2}^{+}\right)$ & ${ }^{6} P_{\frac{7}{2}}\left(J^{P}=\frac{7}{2}^{+}\right)$ & & \\
\hline$\left(Y, I, I_{3}\right)$ & {$\left[\frac{1}{2}_{0}^{-} \otimes 1^{+}\right]_{\frac{3}{2}} \otimes 1^{-}$} & {$\left[\frac{1}{2}_{1}^{-} \otimes 1^{+}\right]_{\frac{3}{2}} \otimes 1^{-}$} & {$\left[\frac{3}{2}_{1}^{-} \otimes 1^{+}\right]_{\frac{3}{2}} \otimes 1^{-}$} & {$\left[\frac{3}{2}_{1}^{-} \otimes 1^{+}\right]_{\frac{5}{2}} \otimes 1^{-}$} & {$\left[\frac{3}{2}_{1}^{-} \otimes 1^{+}\right]_{\frac{5}{2}} \otimes 1^{-}$} & & \\
\hline$\left(1, \frac{1}{2}, \frac{1}{2}\right)$ & 3.259 & 4.069 & 1.960 & 2.753 & 3.666 & & \\
\hline
\end{tabular}

\section{$0.967 \mu_{p}$ respectively.}

Our results clearly show that the magnetic moments of the hidden-charm pentaquarks with different configurations are very different. In other words, the experimental measurement of the magnetic moment of the hidden-charm pentaquark indeed can help distinguish their inner structure.

The $P_{c}$ states can be produced through either the photo-production or electro-production process $\gamma^{(*)} P \rightarrow P_{c} \rightarrow J / \psi P$. In order to extract the magnetic moments of the $P_{c}$ states, one could study one more challenging process $\gamma^{(*)} P \rightarrow P_{c} \rightarrow P_{c} \gamma \rightarrow J / \psi P \gamma$. A very similar process $\gamma^{(*)} P \rightarrow \Delta^{+} \rightarrow \Delta^{+} \gamma \rightarrow \pi^{0} P \gamma$ was used to extract the magnetic moment of the $\Delta$ resonance [39, 40]. The total and differential cross sections may be sensitive to the magnetic moment of the hidden-charm pentaquark, which might be extracted by comparing theoretical predictions with the measured cross sections. We are looking forward to future experimental progress along these directions.

\section{Acknowledgments}

This project is supported by National Natural Science Foundation of China under Grants No. 11222547, No. 11175073, No. 11575008, and 973 program. X.L. is also supported by National Program for Support of Top-notch Youth Professionals.

[1] R. Aaij et al. (LHCb Collaboration), Observation of $J / \psi p$ Resonances Consistent with Pentaquark States in $\Lambda_{b}^{0} \rightarrow J / \psi K^{-} p$ Decays, Phys. Rev. Lett. 115, 072001 (2015). 
[2] R. Aaij et al. (LHCb Collaboration), Model-independent evidence for $J / \psi p$ contributions to $\Lambda_{b}^{0} \rightarrow J / \psi p K^{-}$decays, Phys. Rev. Lett. 117, 082002 (2016).

[3] Z. C. Yang, Z. F. Sun, J. He, X. Liu and S. L. Zhu, The possible hidden-charm molecular baryons composed of anti-charmed meson and charmed baryon, Chin. Phys. C 36, 6 (2012).

[4] J. J. Wu, R. Molina, E. Oset and B. S. Zou, Prediction of narrow $N^{*}$ and $\Lambda^{*}$ resonances with hidden charm above 4 GeV, Phys. Rev. Lett. 105, 232001 (2010).

[5] J. J. Wu, R. Molina, E. Oset and B. S. Zou, Dynamically generated $N^{*}$ and $\Lambda^{*}$ resonances in the hidden charm sector around $4.3 \mathrm{GeV}$, Phys. Rev. C 84, 015202 (2011).

[6] W. L. Wang, F. Huang, Z. Y. Zhang and B. S. Zou, $\Sigma_{c} \bar{D}$ and $\Lambda_{c} \bar{D}$ states in a chiral quark model, Phys. Rev. C 84, 015203 (2011).

[7] H. X. Chen, W. Chen, X. Liu and S. L. Zhu, The hidden-charm pentaquark and tetraquark states, Phys. Rept. 639, 1 (2016).

[8] M. Karliner and J. L. Rosner, New Exotic Meson and Baryon Resonances from Doubly-Heavy Hadronic Molecules, Phys. Rev. Lett. 115, $122001(2015)$

[9] R. Chen, X. Liu, X. Q. Li and S. L. Zhu, Identifying exotic hidden-charm pentaquarks, Phys. Rev. Lett. 115,132002 (2015).

[10] H. X. Chen, W. Chen, X. Liu, T. G. Steele and S. L. Zhu, Towards exotic hidden-charm pentaquarks in QCD, Phys. Rev. Lett. 115, 172001 (2015).

[11] H. Huang, C. Deng, J. Ping and F. Wang, Possible pentaquarks with heavy quarks, arXiv:1510.04648 [hep-ph].

[12] G. Yang and J. Ping, The Structure of Pentaquarks $P_{c}^{+}$in the Chiral Quark Model, arXiv:1511.09053 [hep-ph].

[13] L. Roca, J. Nieves and E. Oset, LHCb pentaquark as a $\bar{D}^{*} \Sigma_{c}-\bar{D}^{*} \Sigma_{c}^{*}$ molecular state, Phys. Rev. D 92,094003 (2015)

[14] J. He, $\bar{D} \Sigma_{c}^{*}$ and $\bar{D}^{*} \Sigma_{c}$ interactions and the LHCb hidden-charmed pentaquarks, Phys. Lett. B 753, 547 (2016).

[15] T. J. Burns, Phenomenology of $\mathrm{P}_{c}(4380)^{+}, \mathrm{P}_{c}(4450)^{+}$and related states, Eur. Phys. J. A 51,152 (2015).

[16] Q. F. L and Y. B. Dong, Strong decay mode $J / \psi p$ of hidden charm pentaquark states $P_{c}^{+}(4380)$ and $P_{c}^{+}(4450)$ in $\Sigma_{c} \bar{D}^{*}$ molecular scenario, Phys. Rev. D 93, 074020 (2016).

[17] R. Chen, X. Liu and S. L. Zhu, Hidden-charm molecular pentaquarks and their charm-strange partners, Nucl. Phys. A 954, 406 (2016).

[18] M. Monemzadeh, N. Tazimiand and S. Babaghodrat, Calculating Masses of Pentaquarks Composed of Baryons and Mesons, Adv. High Energy Phys. 2016, 6480926 (2016).

[19] A. Feijoo, V. K. Magas, A. Ramos and E. Oset, A hidden-charm $S=-1$ pentaquark from the decay of $\Lambda_{b}$ into $J / \psi \eta \Lambda$ states, Eur. Phys. J. C 76, 446 (2016).

[20] D. E. Kahana and S. H. Kahana, LHCb $P_{c}^{+}$Resonances as Molecular States, arXiv:1512.01902 [hep-ph].

[21] C. W. Shen, F. K. Guo, J. J. Xie and B. S. Zou, Disentangling the hadronic molecule nature of the $P_{c}(4380)$ pentaquark-like structure, Nucl. Phys. A 954, 393 (2016).

[22] G. J. Wang, L. Ma, X. Liu and S. L. Zhu, Strong decay patterns of the hidden-charm pentaquark states $P_{c}(4380)$ and $P_{c}(4450)$, Phys. Rev. D 93, 034031 (2016)

[23] L. Maiani, A. D. Polosa and V. Riquer, The New Pentaquarks in the Diquark Model, Phys. Lett. B 749, 289 (2015).

[24] V. V. Anisovich, M. A. Matveev, J. Nyiri, A. V. Sarantsev and A. N. Semenova, Pentaquarks and resonances in the $p J / \psi$ spectrum, arXiv:1507.07652 [hep-ph].

[25] Z. G. Wang, Analysis of $P_{c}(4380)$ and $P_{c}(4450)$ as pentaquark states in the diquark model with QCD sum rules, Eur. Phys. J. C 76, 70 (2016).

[26] G. N. Li, X. G. He and M. He, Some Predictions of Diquark Model for Hidden Charm Pentaquark Discovered at the LHCb, JHEP 1512 (2015) 128.

[27] R. F. Lebed, The Pentaquark Candidates in the Dynamical Diquark Picture, Phys. Lett. B 749, 454 (2015).

[28] R. Zhu and C. F. Qiao, Phys. Lett. B 756, 259 (2016).

[29] H. X. Chen, L. S. Geng, W. H. Liang, E. Oset, E. Wang and J. J. Xie, Looking for a hidden-charm pentaquark state with strangeness $S=-1$ from $\Xi_{b}^{-}$decay into $J / \Psi K^{-} \Lambda$, Phys. Rev. C 93, 065203 (2016).

[30] R. F. Lebed, Do the $P_{c}^{+}$Pentaquarks Have Strange Siblings? Phys. Rev. D 92, 114030 (2015).

[31] Q. F. L, X. Y. Wang, J. J. Xie, X. R. Chen and Y. B. Dong, Neutral hidden charm pentaquark states $P_{c}^{0}(4380)$ and $P_{c}^{0}(4450)$ in $\pi^{-} p \rightarrow J / \psi n$ reaction, Phys. Rev. D 93, 034009 (2016).

[32] X. H. Liu, Q. Wang and Q. Zhao, Understanding the newly observed heavy pentaquark candidates, Phys. Lett. B 757, 231 (2016).

[33] M. Mikhasenko, A triangle singularity and the LHCb pentaquarks, arXiv:1507.06552 [hep-ph].

[34] F. K. Guo, U. G. Meiner, W. Wang and Z. Yang, How to reveal the exotic nature of the $\mathrm{P}_{c}(4450)$, Phys. Rev. D 92, 071502 (2015).

[35] N. N. Scoccola, D. O. Riska and M. Rho, Pentaquark candidates $P_{c}^{+}(4380)$ and $P_{c}^{+}(4450)$ within the soliton picture of baryons, Phys. Rev. D 92, 051501 (2015).

[36] Y. R. Liu, P. Z. Huang, W. Z. Deng, X. L. Chen and S. L. Zhu, Pentaquark magnetic moments in different models, Phys. Rev. C 69, 035205 (2004).

[37] K. A. Olive et al. [Particle Data Group Collaboration], Review of Particle Physics, Chin. Phys. C 38, 090001 (2014).

[38] D. Ebert, R. N. Faustov and V. O. Galkin, Masses of tetraquarks with open charm and bottom, Phys. Lett. B 696, 241 (2011).

[39] V. Pascalutsa and M. Vanderhaeghen, Magnetic moment of the Delta(1232)-resonance in chiral effective field theory, Phys. Rev. Lett. 94, 102003 (2005).

[40] V. Pascalutsa and M. Vanderhaeghen, Chiral effective-field theory in the Delta(1232) region. II. Radiative pion photoproduction, Phys. Rev. D 77, 014027 (2008). 